ARTICLE

https://doi.org/10.1038/s41467-019-09118-9

\title{
Soluble TREM2 ameliorates pathological phenotypes by modulating microglial functions in an Alzheimer's disease model
}

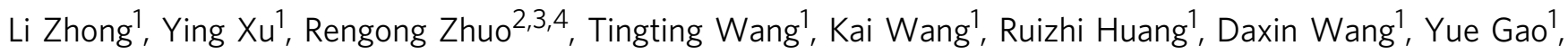

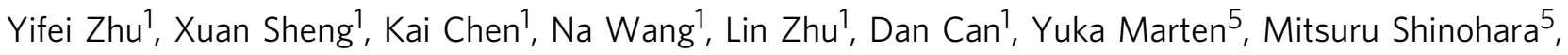

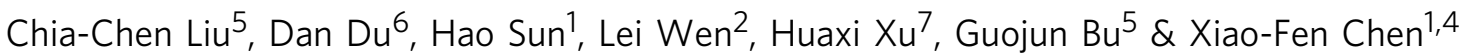

Triggering receptor expressed on myeloid cells 2 (TREM2) is a microglial surface receptor genetically linked to the risk for Alzheimer's disease (AD). A proteolytic product, soluble TREM2 (sTREM2), is abundant in the cerebrospinal fluid and its levels positively correlate with neuronal injury markers. To gain insights into the pathological roles of sTREM2, we studied STREM 2 in the brain of $5 \times F A D$ mice, a model of $A D$, by direct stereotaxic injection of recombinant STREM2 protein or by adeno-associated virus (AAV)-mediated expression. We found that STREM2 reduces amyloid plaque load and rescues functional deficits of spatial memory and long-term potentiation. Importantly, sTREM2 enhances microglial proliferation, migration, clustering in the vicinity of amyloid plaques and the uptake and degradation of A $\beta$. Depletion of microglia abolishes the neuroprotective effects of STREM2. Our study demonstrates a protective role of STREM2 against amyloid pathology and related toxicity and suggests that increasing sTREM2 can be explored for AD therapy.

\footnotetext{
${ }^{1}$ Fujian Provincial Key Laboratory of Neurodegenerative Disease and Aging Research, Institute of Neuroscience, School of Medicine, Xiamen University, Xiamen 361102, China. ${ }^{2}$ Department of Traditional Chinese Medicine, School of Medicine, Xiamen University, Xiamen 361102, China. ${ }^{3}$ Xiamen Key Laboratory of Chiral Drugs, School of Medicine, Xiamen University, Xiamen 361102, China. ${ }^{4}$ Shenzhen Research Institute of Xiamen University, Shenzhen 518063, China. ${ }^{5}$ Department of Neuroscience, Mayo Clinic, Jacksonville, FL 32224, USA. ${ }^{6}$ School of Medicine, Xiamen University, Xiamen 361102 , China. ${ }^{7}$ Neuroscience Initiative, Sanford-Burnham-Prebys Medical Discovery Institute, La Jolla, CA 92037, USA. These authors contributed equally: Li Zhong, Ying Xu. Correspondence and requests for materials should be addressed to G.B. (email: bu.guojun@mayo.edu) or to X.-F.C. (email: chenxf@xmu.edu.cn)
} 
A lzheimer's disease $(\mathrm{AD})$ is a progressive neurodegenerative disorder and the most prominent cause of dementia in the elderly population. The histopathological hallmarks of $\mathrm{AD}$ include the parenchymal deposition of amyloid- $\beta$ ( $\mathrm{A} \beta$ ) plaques, the formation of tau neurofibrillary tangles, and neuroinflammation ${ }^{1,2}$. With the accumulation of these lesions in the brain, AD patients ultimately suffer from synaptic loss, neuronal death, and cognitive decline ${ }^{3}$. Although the pathogenic events for $\mathrm{AD}$ are likely multifactorial, genetic, pathological, and functional studies suggest that the disequilibrium between $A \beta$ production and clearance is a contributing and perhaps a driving event leading to $\mathrm{AD}^{4}$. At present, enhancing the clearance of $\mathrm{A} \beta$ remains an attractive therapeutic or preventive strategy for $\mathrm{AD}^{5}$. Microglia are effective phagocytes in the central nervous system (CNS) for the uptake and proteolytic clearance of both soluble and fibrillary forms of $A \beta^{6}$. Early studies have found that microglial cells are clustered around amyloid plaques in the brain of $\mathrm{AD}$ patients ${ }^{7,8}$ and $\mathrm{AD}$ mouse models ${ }^{9}$. Recently, plaqueassociated microglia have been shown to constitute a protective barrier that compacts amyloid fibrils and reduces their toxicity ${ }^{10,11}$. The roles of microglia in $\mathrm{AD}$ have been further highlighted by recent genetic studies showing rare coding variants in several genes highly expressed in microglia as risk factors for late-onset $\mathrm{AD}^{12-19}$. Among them, a loss-of-function $\mathrm{R} 47 \mathrm{H}$ mutation in the triggering receptor expressed on myeloid cells 2 (TREM2) constitutes one of the strongest single-allele genetic risk factors for $\mathrm{AD}^{13,15,16}$.

TREM2 is a type I transmembrane innate immune receptor predominantly expressed by microglia within the $\mathrm{CNS}^{20,21}$. The ectodomain of TREM2 has been reported to bind anionic and zwitterionic lipids ${ }^{22}$, apolipoproteins (including apoE, apoJ, and apoA) and lipoprotein particles ${ }^{23-25}$, and oligomeric $\mathrm{A} \beta$ as reported recently 26,27 . In the disease context, TREM2 plays important roles in microglial phagocytosis of apoptotic neurons, damaged myelin, and amyloid plaques ${ }^{21,28}$. TREM2 has also been shown to be essential for synaptic pruning in early development $^{29}$. Furthermore, TREM2 regulates microglial biosynthetic metabolism $^{30}$, proliferation ${ }^{31}$, survival ${ }^{31}$, cytokine release ${ }^{32}$, and their accumulation around plaques ${ }^{11}$. The impact of TREM2 on plaque accumulation was examined in Trem2-deficient AD mouse models, but the results were conflicting ${ }^{22,33}$. Interestingly, a later study suggested disease progression-dependent effects of TREM2 on amyloid pathology by demonstrating that Trem2 deficiency ameliorates amyloid pathology early, but exacerbates it late in the disease process ${ }^{34}$.

TREM2 undergoes regulated proteolytic cleavage by ADAM10 and ADAM17 at H157-S158 peptide bond, resulting in the liberation of soluble TREM2 (sTREM2) ${ }^{35-37}$. sTREM2 is abundantly detected in human cerebrospinal fluid (CSF) and its levels are elevated in the CSF of patients with sporadic $\mathrm{AD}^{38-41}$. Interestingly, the levels of sTREM2 change dynamically during the progression of $\mathrm{AD}$, peaking at the early symptomatic stages of the disease ${ }^{38,40}$. Importantly, the CSF concentrations of sTREM2 correlate with neuronal injury markers, including the CSF levels of total tau and phospho-tau; thus, they may serve as an immunomodulatory biomarker for neurodegeneration. Remarkably, sTREM2 was found to co-localize with neurons and plaques in vivo ${ }^{42}$, with functions remaining to be determined. We have recently reported that sTREM2 exerts functional roles in microglia by promoting inflammatory responses and shielding them from apoptosis ${ }^{43}$. However, whether sTREM2 also has protective effects against amyloid pathology and the related synaptic toxicity remains to be defined. In this study, we explored the effects of sTREM 2 on pathological phenotypes in $5 \times$ FAD mouse model by direct stereotaxic injection of recombinant sTREM2 protein or by an adenovirus-associated virus (AAV)-mediated expression strategy. We found that sTREM2 reduces amyloid- $\beta$ (A $\beta$ ) pathology and improves cognitive and synaptic functions by modulating microglial activity in $5 \times \mathrm{FAD}$ mouse model, thus providing a rationale that sTREM2 can be explored for $\mathrm{AD}$ therapy.

\section{Results}

sTREM2 reduces plaque load and the associated toxicity. We had previously developed a mammalian expression and purification system for recombinant sTREM2 protein (Supplementary Fig. 1a) ${ }^{43}$. To assess the impact of sTREM2 on AD-related pathology, we injected a recombinant sTREM2 protein into the right hippocampus of $5 \times \mathrm{FAD}$ mice, with phosphate-buffered saline (PBS) injected into the left hippocampus as a vehicle control (Fig. 1a). Twenty-four hours after stereotaxic injection of sTREM2, immunofluorescence staining with human TREM2 antibody showed a sTREM2 distribution pattern that is diffused but limited to the right hippocampus (Supplementary Fig. 1b). However, the sTREM2 signal decayed thereafter and was barely detectable on day 3 and 7 after injection. Seven days after delivery to the hippocampus of $5 \times \mathrm{FAD}$ mice, sTREM 2 dramatically reduced the amyloid plaque load in the ipsilateral hippocampus (Fig. 1b, c). Of note, the number of large plaques $(>40 \mu \mathrm{m}$ in diameter) was markedly reduced by sTREM2 $(P<0.0001$, paired Student's $t$ test) (Fig. 1d).

In addition to the amount of $\mathrm{A} \beta$ accumulation, the degree of plaque compaction plays a crucial role in determining the neurotoxicity of the plaque. It has been suggested that microglia clustered around the amyloid plaque constitute a barrier which impacts plaque composition and toxicity ${ }^{10}$. To gain insight into the effects of sTREM2 on microglial barrier function, we quantified the number of microglia in the vicinity of an amyloid plaque and found a significant increase upon sTREM2 administration (Fig. 1e, f). Consistent with its protective effects on plaque deposition, sTREM2 significantly reduced the total area of dystrophic neurites, as detected by immunostaining with an antibody against Lamp1 (Fig. 1g, h). However, no significant differences were observed between vehicle and sTREM2 treatment when calculating the amount of dystrophic neurites per plaque (Fig. 1i). Thus, distinct from the full-length TREM2, sTREM2 is unlikely involved in compacting amyloid fibrils to reduce their toxicity ${ }^{11}$. Collectively, these data reveal that sTREM2 protein reduces amyloid plaque deposition and enhances the clustering of microglia in the vicinity of the plaque, thereby reducing plaque load and the plaque-associated neurotoxicity.

sTREM2 promotes microglial proliferation and migration. Proliferation and activation of microglia around the sites of amyloid plaque deposition is a prominent feature of $\mathrm{AD}^{44}$. The ionized calcium-binding adapter molecule 1 (Iba1) is widely employed as an immunohistochemical marker for both ramified and activated microglia, and its expression is increased upon microglial activation $^{45,46}$. We found that the expression of Ibal in the hippocampus of $5 \times \mathrm{FAD}$ mice was significantly increased at the protein level, and a trend toward increased mRNA level upon sTREM2 administration (Fig. 2a-c). However, no significant differences were detected for the astrocytic marker GFAP. Staining of brain sections further revealed that sTREM2 administration led to a significantly higher number of proliferating microglia $\left(\mathrm{Iba}^{+} / \mathrm{Ki} 67^{+}\right)$ and the total number of microglia $\left(\mathrm{Ibal}^{+}\right)$in the hippocampus (Fig. 2d-f). Interestingly, the increased Ibal staining induced by sTREM2 on day 1 was sustained throughout day 7 , even though sTREM2 was barely detectable on day 3 and 7 after injection (Supplementary Fig. 1b). In addition to Iba1, the expression levels of 

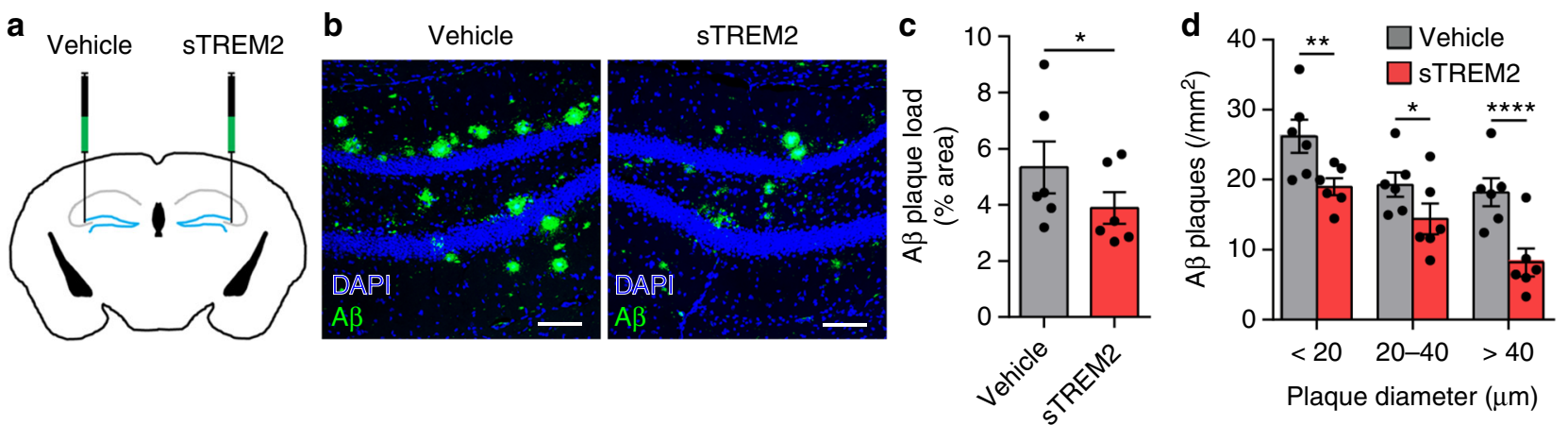

e

$\mathbf{f}$
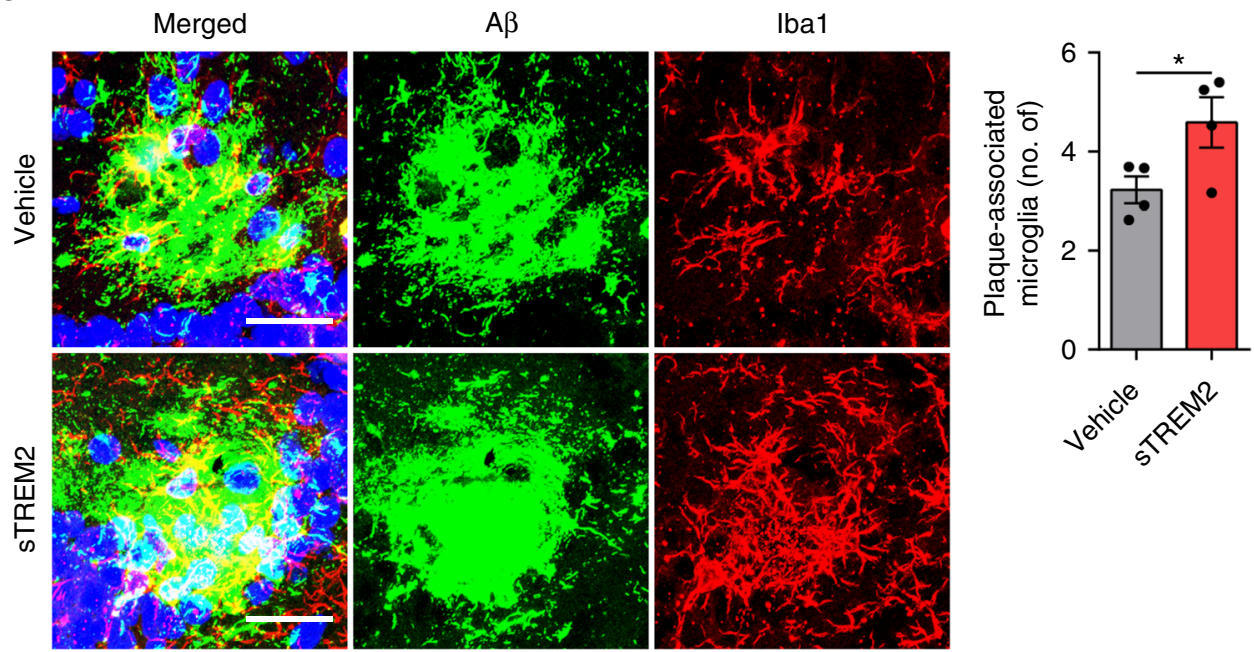

h

Plaque diameter $(\mu \mathrm{m})$
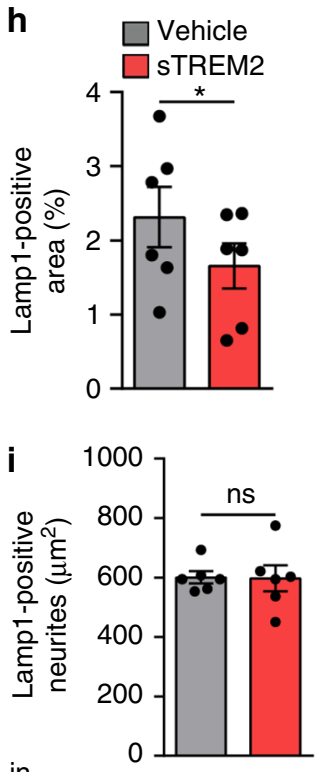

g $A \beta$

lba1

Lamp1

Merged

Zoom in
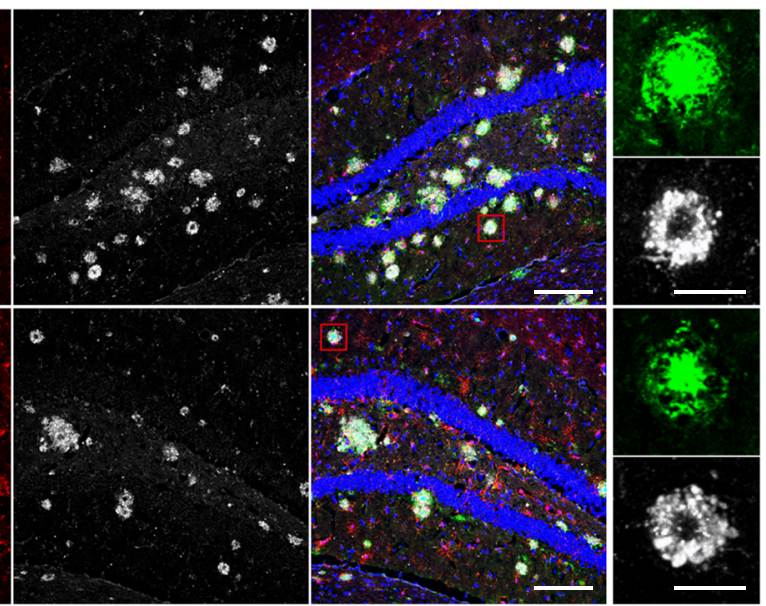

pro-inflammatory cytokines, including IL-1 $\beta$ and TNF, were significantly increased in the presence of sTREM2 (Supplementary Fig. 2a), consistent with our previous report that sTREM2 promotes microglial activation ${ }^{43}$. Importantly, both the pro-inflammatory cytokine IL-1 $\beta$ and the immune-activating molecule lipopolysaccharide enhanced the production of sTREM2 in primary microglia, as measured by enzyme-linked immunosorbent assay (ELISA); while the anti-inflammatory cytokine IL-10 had a minimal effect (Supplementary Fig. 2b). Therefore, sTREM2 and the proinflammatory cytokines appear to form a positive feedback loop, resulting in sustained microglial activation.
Since sTREM2 increases the number of plaque-associated microglia, we further tested a hypothesis that sTREM2 might act as a chemoattractant to promote microglial migration. Primary microglia isolated from wild-type (WT) mice were subjected to the transwell migration assay in the presence or absence of sTREM2. Interestingly, treatment with native but not heat-inactivated sTREM2 led to increased migration of microglia, as compared with the vehicle control (Fig. 2g, h). Our data hence suggest that sTREM2 not only enhances microgliosis in $5 \times \mathrm{FAD}$ mice, but also acts as a chemoattractant for microglia. 
Fig. 1 sTREM2 reduces amyloid plaque deposition and increases the number of plaque-associated microglia. a The 5×FAD mice at 7-month-old age were injected with vehicle (PBS) in the left hemisphere and $6 \mu \mathrm{g}$ of sTREM2 protein in the right hemisphere. $\mathbf{b}$ Coronal sections of vehicle- or sTREM2-injected $5 \times$ FAD mice were stained with DAPI (blue) for nuclei and MOAB-2 (green) for A $\beta$. Representative z-stack images of the hippocampus regions are shown. Original magnification $\times 20$; scale bar, $100 \mu \mathrm{m}$. c Quantitation of amyloid plaque deposition in $\mathbf{b}(n=6$ mice, 28 fields of each group for analysis, paired Student's $t$ test). d Quantitation of the number of plaques with different sizes in $\mathbf{b}$ ( $n=6$ mice, 28 fields of each group for analysis, paired Student's $t$ test). e Coronal sections from sTREM2-injected 5×FAD mice were stained with DAPI (blue) for nuclei, MOAB-2 (green) for A $\beta$, and lba1 (red) for microglia. Representative z-stack images of the hippocampus regions are shown. Scale bar, $25 \mu \mathrm{m}$. $\mathbf{f}$ Quantitation of the number of plaque-associated microglia in e $(n=4$ mice, 58 plaques of vehicle and 41 plaques of sTREM2 for analysis, paired Student's $t$ test). Plaques with $50 \mu \mathrm{m}$ in diameter were selected for analysis. $\mathbf{g}$ Coronal sections from sTREM2-injected 5×FAD mice were stained with DAPI (blue) for nuclei, MOAB-2 (green) for A $\beta$, Iba1 (red) for microglia, and Lamp1 (white) for dystrophic neurites. Representative $z$-stack images of the hippocampus regions are shown. Original magnification $\times 20$; scale bar, $100 \mu \mathrm{m}$. Zoom-in images on the right with a scale bar equal to $25 \mu \mathrm{m}$. $\mathbf{h}$ Quantitation of the total area of Lamp1-positive dystrophic neurites in $\mathbf{g}(n=6$ mice, 20 fields of each group for analysis, paired Student's $t$ test). i Quantitation of the area of Lamp1-positive dystrophic neurites within each plaque in $\mathbf{g}$ ( $n=6$ mice, 40 plaques of vehicle and 41 plaques of STREM2 for analysis, paired Student's $t$ test). All data are presented as mean \pm SEM ${ }^{\star} p<0.05 ;{ }^{\star \star} p<0.01 ;{ }^{\star \star \star \star} p<0.0001 ;$ ns, not significant

sTREM2 promotes microglial phagocytosis and clearance of A $\boldsymbol{\beta}$. Microglia clustering in the vicinity of plaques is interpreted as an attempt to clear the pathological deposits of $A \beta$ through phagocytosis and degradation. Indeed, quantitative assessment of the area of colocalization of $\mathrm{A} \beta$ with $\mathrm{CD} 68$, a phagocytic marker for microglia in the brain, revealed a significant increase in sTREM2-injected $5 \times$ FAD mice (Fig. 3a, b), suggesting that sTREM2 likely promotes phagocytic $\mathrm{A} \beta$ uptake by microglia. To further test this in vivo, we administered intraperitoneally methoxy-X04 $3 \mathrm{~h}$ prior to microglial isolation and analyzed the proportion of methoxy-X04-positive microglial cells by flow cytometry. Comparing PBS-injected WT mice and $5 \times \mathrm{FAD}$ mice treated with native or heat-inactivated sTREM2 revealed a significant increase in the number of $\mathrm{CD}_{11 \mathrm{~b}^{+}} \mathrm{CD} 45^{\text {low }}$ resident microglia in sTREM2administered $5 \times \mathrm{FAD}$ mice, while no significant change was observed for the number of $\mathrm{CD} 11 \mathrm{~b}^{+} \mathrm{CD} 45^{\text {high }}$ peripheral macrophage (Fig. 3c, d). Therefore, the increased number of Ibal-positive cells upon sTREM2 treatment is likely contributed by resident microglia rather than infiltrating macrophages. A significant increase in the proportion of methoxy-X04-positive microglial cells was observed for $5 \times \mathrm{FAD}$ mice injected with native sTREM2 (Fig. 3e, f), further demonstrating that sTREM2 enhances microglial phagocytosis of $A \beta$ in vivo.

We further examined the effects of sTREM2 on A $\beta$ uptake and/ or degradation in cultured microglial cells. Primary postnatal microglia isolated from WT mice were incubated with fluorescently FAM-labeled A $\beta 42$ (FAM-A $\beta 42)$. We found an increase in FAM-A $\beta 42$ uptake upon sTREM2 stimulation (Fig. $3 \mathrm{~g}, \mathrm{~h}$ ). However, the effect was diminished in the presence of a phagocytosis inhibitor cytochalasin $\mathrm{D}$. To further investigate the role of sTREM2 in microglial $A \beta$ endocytic trafficking, we incubated microglial cells with $A \beta$, together with vehicle control or sTREM2 in the presence or absence of lysosomal enzyme inhibitors (Pepstatin A, Leupeptin, and E-64d) or chloroquine. Cell-associated $A \beta$ levels were significantly increased by sTREM2 in the presence of lysosomal inhibitors (Fig. $3 \mathrm{i}$ and Supplementary Fig. 3a). When lysosomal degradation of $A \beta$ was calculated by subtracting the cell-associated $A \beta$ in the absence of inhibitors from that in the presence of inhibitors, we found that sTREM2 significantly enhanced A $\beta$ lysosomal degradation, as compared with vehicle control (Fig. $3 \mathrm{j}$ and Supplementary Fig. 3b).

Since the steady-state levels of brain $\mathrm{A} \beta$ represent a dynamic equilibrium between its rate of clearance and production from the amyloid precursor protein (APP), we further explored the molecular mechanism by which sTREM 2 reduces A $\beta$ levels. We performed a series of experiments to evaluate whether sTREM2 induces changes in the levels of APP, its cleavage products, or $\mathrm{A} \beta$-degrading enzymes in sTREM2-injected $5 \times \mathrm{FAD}$ mice. No significant changes in the levels of full-length APP, a-CTF, or $\beta$-CTF were detected upon sTREM2 injection in $5 \times \mathrm{FAD}$ mice (Supplementary Fig. 3c, d). Quantitative real-time polymerase chain reaction (PCR) of the transcript levels of $\mathrm{A} \beta$-degrading enzymes, including insulin-degrading enzyme (IDE), neprilysin (NEP), endothelin-converting enzyme 1 (ECE1), matrix metalloproteinase 2 (MMP2), and matrix metalloproteinase 9 (MMP9), did not detect any significant alterations in response to sTREM2 treatment (Supplementary Fig. 3e). Taken together, we provide both in vivo and in vitro evidence that sTREM2 promotes microglial phagocytosis and clearance of $A \beta$, which may account for sTREM2-mediated reduction of $A \beta$ deposition in the brain of $5 \times \mathrm{FAD}$ mice.

Microglia are essential for the protective effects of sTREM2. Both our current and previous studies pinpoint a critical role of sTREM2 in modulating microglial function ${ }^{43}$. To determine whether microglia mediate the protective effects of sTREM2 on amyloid pathology, we employed the CSF1R inhibitor PLX3397 to deplete microglia from $5 \times \mathrm{FAD}$ mice ${ }^{47,48}$. Following 14 days of PLX3397 treatment, microglial number as assessed by Ibal staining was substantially reduced, with efficiency comparable with the previous work in $5 \times \mathrm{FAD}$ mice (Supplementary Fig. $4 a, b)^{48}$. Consistent with previous reports that microglial elimination in $5 \times \mathrm{FAD}$ mice prevents neuronal loss ${ }^{48}$, increased levels of synaptic markers (including NR2A, NR1, GluR1, and vGluT1) were observed in PLX3397 administered to $5 \times$ FAD mice (Supplementary Fig. 4c, d). However, no significant changes in the levels of full-length APP, $a-C T F$, or $\beta$-CTF were detected upon PLX3397 treatment. To determine if microglia are responsible for sTREM2-mediated plaque reduction, we depleted microglia with 14 days of PLX3397 treatment, followed by administering sTREM2 to the hippocampus of $5 \times \mathrm{FAD}$ mice (Fig. 4a). In contrast to the control without PLX3397 treatment, the plaque load was no longer reduced by sTREM 2 in $5 \times F A D$ mice treated with PLX3397, suggesting an indispensable role of microglia in sTREM2-mediated plaque reduction (Fig. 4b, c).

Next, we studied the impact of sTREM2 administration on hippocampal synaptic plasticity by inducing long-term potentiation (LTP) in the Schaffer collateral (SC) pathway with highfrequency stimulation. Intriguingly, the LTP impairment of $5 \times \mathrm{FAD}$ mice was ameliorated when acute hippocampal slices obtained from $5 \times \mathrm{FAD}$ mice were pre-incubated with sTREM2 protein as compared with the vehicle control (Fig. 4d, f). More interestingly, such effects were diminished in microgliadepleted $5 \times$ FAD mice (Fig. 4e, f), suggesting that microglia mediate the protective functions of sTREM2 in synaptic plasticity. In contrast to the $5 \times \mathrm{FAD}$ mice, sTREM 2 had 
a

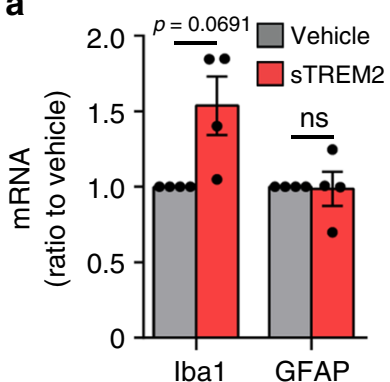

b

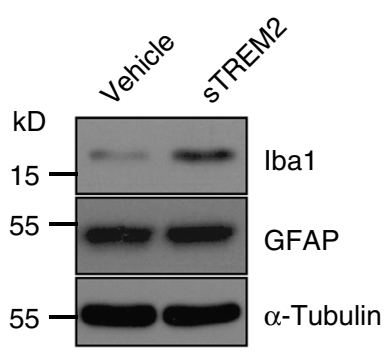

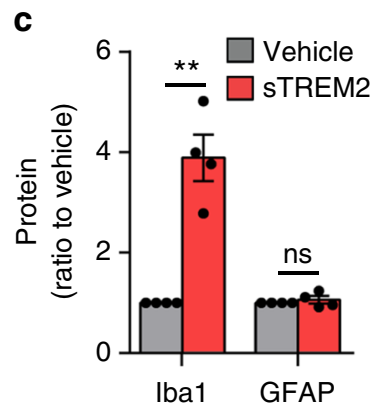

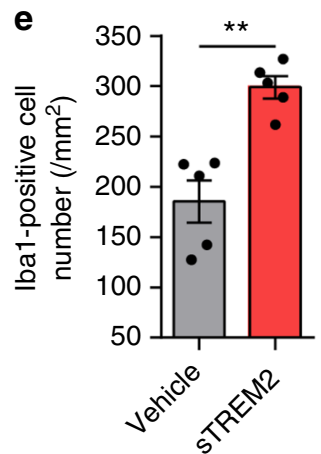

d
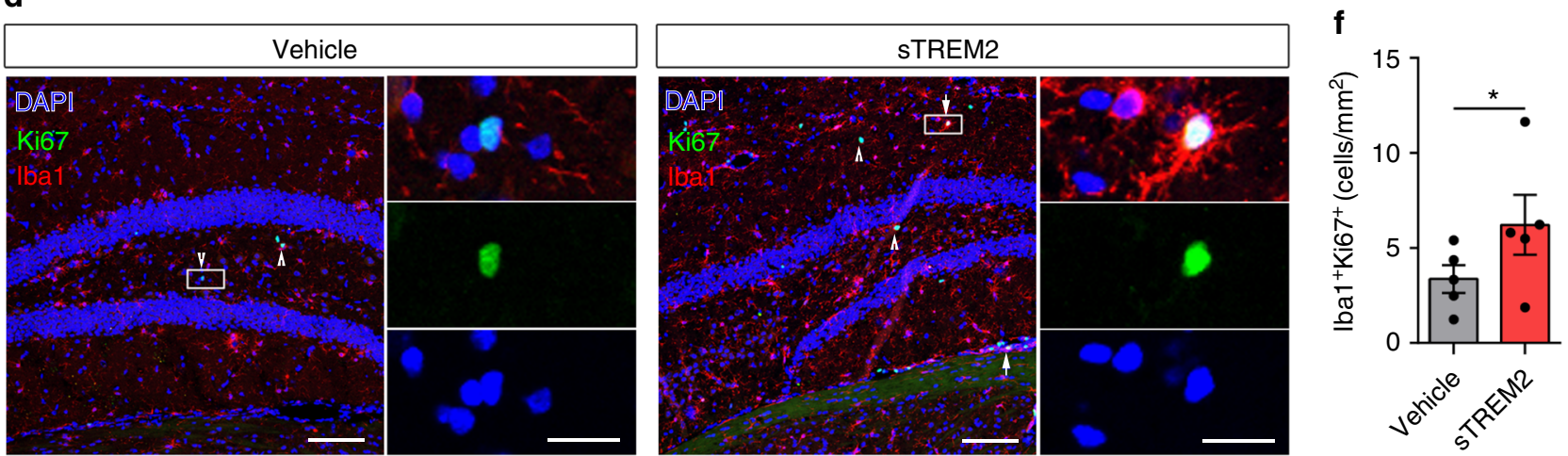

g

Vehicle

STREM2 Inactive sTREM2
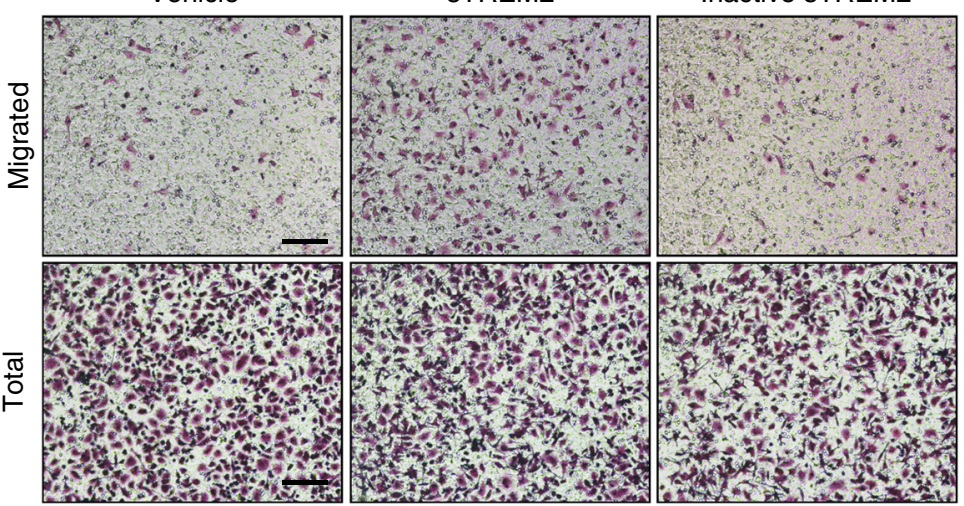

$\mathbf{h}$

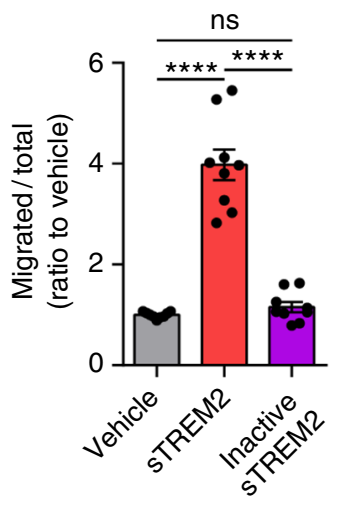

Fig. 2 sTREM2 promotes microgliosis in 5×FAD mice and microglial migration. a The hippocampus was dissected from vehicle- or sTREM2-injected 5×FAD mice. After RNA extraction, the relative mRNA levels of Iba1 and GFAP in the hippocampus shown as a bar graph were determined by quantitative realtime PCR. $\beta$-actin was used as an internal control ( $n=4$ mice per group, paired Student's $t$ test). $\mathbf{b}$ lba1 and GFAP proteins were analyzed by Western blotting 7 days after sTREM2 injection to the hippocampus of 5×FAD mice. $\mathbf{c}$ Quantitation of the protein levels of lba1 and GFAP in $\mathbf{b}$ ( $n=4$ mice per group, paired Student's $t$ test). d Coronal sections from injected 5×FAD mice were stained with DAPI (blue) for nuclei, Ki67 (green) for proliferating cells, and Iba1 (red) for microglia. Representative z-stack images of the hippocampus regions are shown. Original magnification $\times 20 ;$ scale bar, $100 \mu \mathrm{m}$. Images on the right represent enlarged Ki67-positive cells with a scale bar equal to $20 \mu \mathrm{m}$. e Quantitation of the number of Iba1-positive cells in $\mathbf{d}$ ( $n=5 \mathrm{mice}, 22$ fields of each group for analysis, paired Student's $t$ test). $\mathbf{f}$ Quantitation of the number of proliferating microglial cells, as indicated by the co-staining of Ki67 and Iba1 $\left(\mathrm{Iba} 1^{+} \mathrm{Ki} 67^{+}\right)\left(n=5\right.$ mice, 22 fields of each group for analysis, paired Student's $t$ test). $\mathbf{g}$ Primary microglial cells $\left(10^{5}\right)$ from WT mice were plated onto transwell chamber inserts. Following 24-h incubation with vehicle (PBS), native sTREM2 protein (100 nM), or heat-inactivated sTREM2 protein $(100 \mathrm{nM})$, cells migrated through the membrane were stained with hematoxylin and eosin and imaged under a Nikon inverted microscope. Scale bar, $100 \mu \mathrm{m}$. $\mathbf{h}$ Quantitation of the number of migrated cells ( $n=9$ from three independent experiments, one-way ANOVA). All data are presented as mean \pm SEM. ${ }^{\star} p<0.05 ;{ }^{\star \star} p<0.01 ;{ }^{\star \star \star \star} p<0.0001 ;$ ns, not significant

minimal effects on the LTP in either microglia-intact or microglia-eliminated WT mice (Supplementary Fig. 4e-g). To better understand synaptic alterations upon sTREM2 administration, we examined the levels of various proteins involved in synaptic plasticity and function. The levels of postsynaptic glutamate (AMPAR) receptor subunits (GluR1 and GluR2) and the presynaptic vesicular glutamate transporter 1 (vGluT1) were significantly increased upon sTREM2 treatment in the hippocampus (Fig. 4g, h). However, sTREM2 could no longer increase the levels of these proteins in the absence of microglia (Fig. 4i, j).

Recently, sTREM2 was found to co-localize with neurons in vivo ${ }^{42}$. To further investigate whether neurons directly respond to sTREM2, primary neuronal cultures at DIV 12-14 were incubated with sTREM2 and subjected to whole-cell patch-clamp experiments to record miniature excitatory postsynaptic currents 
a

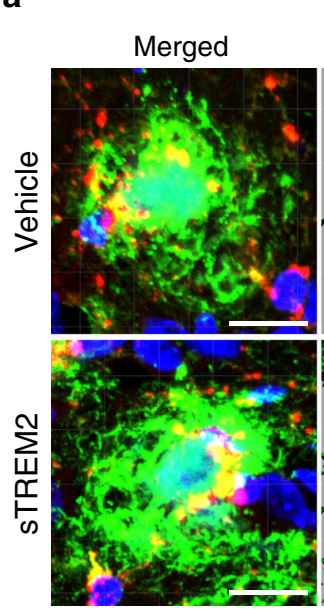

C

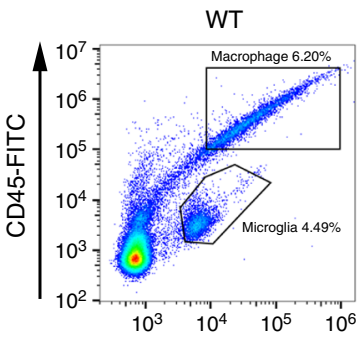

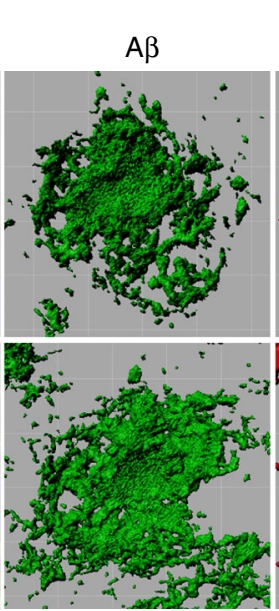

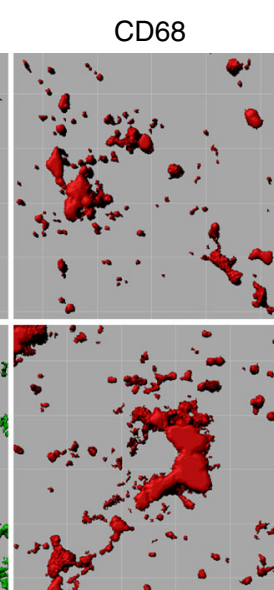

$5 \times F A D$

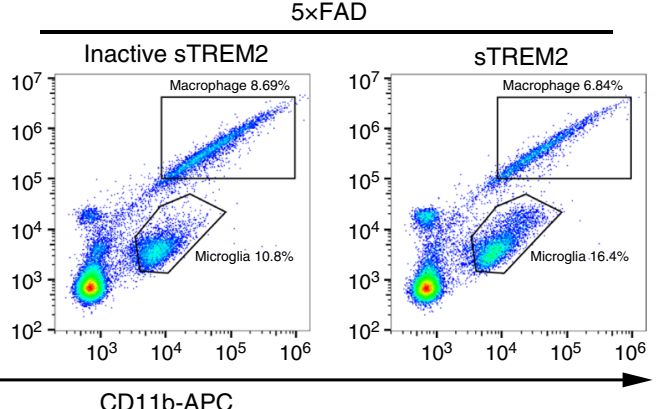

e
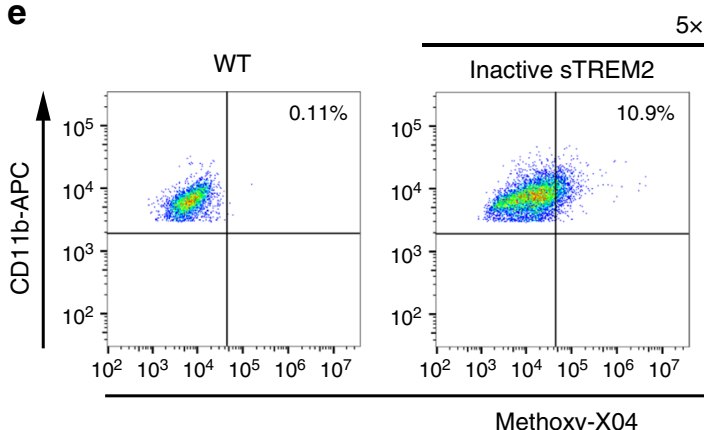

Methoxy-X04

g

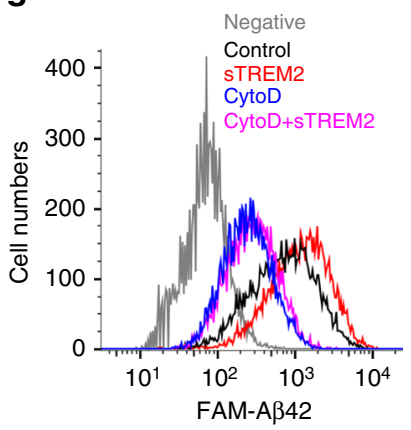

h

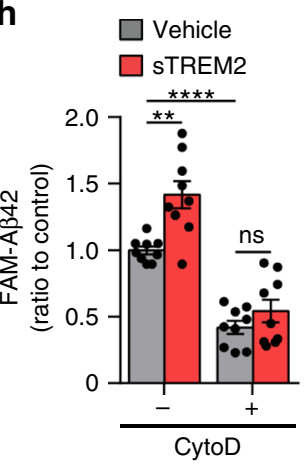

$A \beta$ colocalized with CD68

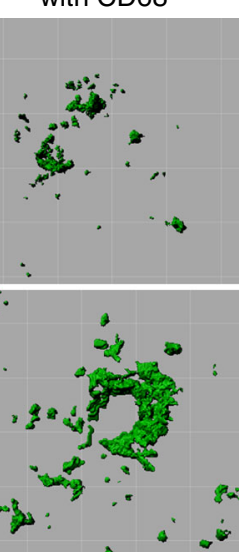

b

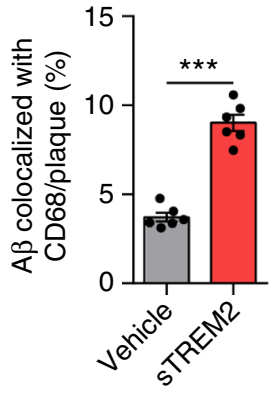

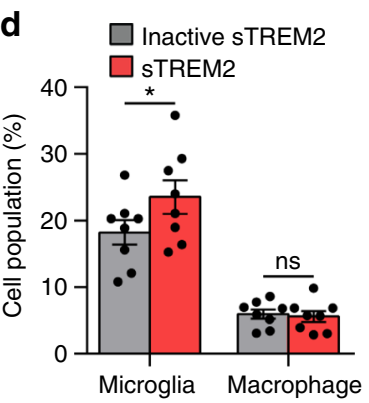

f

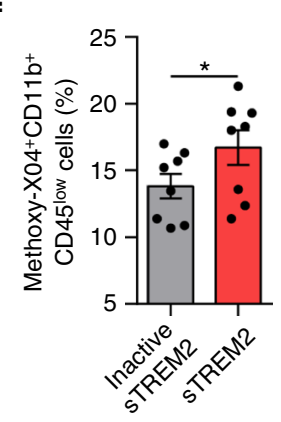

i

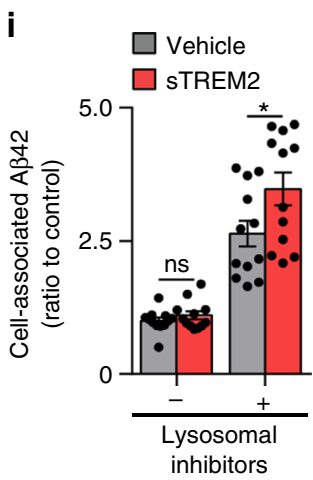

$\mathbf{j}$

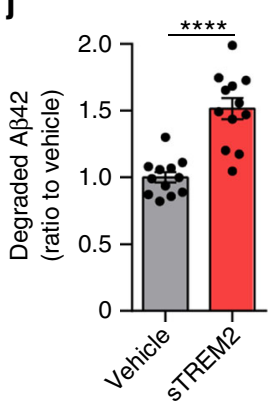

(mEPSCs) and miniature inhibitory postsynaptic currents (mIPSCs). The amplitude and frequency of both mEPSCs and mIPSCs did not significantly differ between Fc- and sTREM-Fctreated groups (Supplementary Fig. 4h-k). Furthermore, sTREM2 did not affect the abundance of synaptic proteins in the primary neuronal cultures (Supplementary Fig. 41, m), suggesting that sTREM2 does not directly modulate synaptic plasticity and function. Taken together, these data indicate that microglia play an indispensable role in mediating the protective effects of sTREM2 on amyloid plaque pathology and synaptic plasticity.

Validation with physiologically produced sTREM2. At the time we started this project, the precise TREM2 cleavage site to 
Fig. 3 STREM2 enhances microglial phagocytosis and degradation of $A \beta$. a Coronal sections from sTREM2-injected $5 \times F A D$ mice were stained with DAPI (blue) for nuclei, MOAB-2 (green) for $A \beta$, or CD68 (red) for a phagocytic phenotype of microglia. Three-dimensional reconstruction of $A \beta$, CD68, and $A \beta$ colocalized with CD68 was shown. Scale bar, $25 \mu \mathrm{m}$. $\mathbf{b}$ Quantitation of the ratio of A $\beta$ colocalized with CD68 within each plaque ( $n=6$ mice, 42 plaques of vehicle and 30 plaques of STREM2 for analysis, paired Student's $t$ test). c Representative FACS dot plots of microglia or macrophage from WT or $5 \times F A D$ mice are shown. Microglia and microphage were identified as CD11b+CD45 low and CD11b+CD45 high, respectively. $\mathbf{d}$ Quantitation of the cell population of microglia or macrophage in $\mathbf{c}(n=8$ mice, paired Student's $t$ test). e Representative FACS dot plots showing the microglial phagocytosis of A $\beta$ in $5 \times F A D$ mice. $\mathbf{f}$ Quantification of the percentage (\%) of methoxy-X04+CD11b+CD45low hippocampal microglial cells by flow cytometry isolated from sTREM2injected 5×FAD mice ( $n=8$ mice, paired Student's $t$ test). $\mathbf{g}$ Representative histogram displaying background fluorescence (negative) of untreated microglia and the distribution of FAM-A 42 -positive cells treated with vehicle or sTREM2 protein. $\mathbf{h}$ Quantitation of the mean fluorescence in $\mathbf{g}$ to indicate the amount of FAM-A $\beta 42$ uptake. Values were normalized to the vehicle control without cyto $D$ ( $n=9$ from three independent experiments, two-way ANOVA, Bonferonni post hoc analyses). $\mathbf{i}$ The amounts of cell-associated A 42 were quantified by ELISA ( $n=12$ from four independent experiments, two-way ANOVA, Bonferonni post hoc analyses). $\mathbf{j}$ The amounts of degraded $A \beta 42$ were calculated by subtracting the cell-associated $A \beta$ in the absence of inhibitors from that in the presence of inhibitors $(n=12$ from four independent experiments, unpaired Student's $t$ test). All data are presented as mean \pm SEM. ${ }^{\star} p<0.05 ;{ }^{\star \star} p<0.01 ;{ }^{\star \star \star} p<0.001 ;{ }^{\star \star \star \star} p<0.0001 ; \mathrm{ns}$, not significant

generate sTREM2 was unknown. More recently, it was reported that TREM2 undergoes regulated shedding at the H157-S158 peptide bond, resulting in the liberation of soluble TREM2 ending at position 157 (sTREM2-157) $35-37$. Hence, we went on to test whether the physiological form of sTREM2-157 protein recapitulates the functions that we defined for the sTREM2-171 mimics (Fig. 5a). Consistent with our previous findings ${ }^{43}$, the sTREM2157 protein was as efficient as the sTREM-171 in both suppressing microglial apoptosis and stimulating the production of inflammatory cytokines (Fig. 5b, c). Furthermore, the native sTREM2-157 protein but not its heat-inactivated form significantly stimulated the migration of microglial cells, consistent with the role of sTREM2-171 protein as a chemoattractant for microglia (Fig. 5d, e). Importantly, the sTREM2-157 protein largely and significantly decreased the deposition of amyloid plaques and promoted the clustering of microglia in the vicinity of plaques (Fig. 5f-i). However, sTREM2 lost these protective functions upon heat inactivation, indicating that the native protein structure is required for sTREM2 function (Supplementary Fig. 5). We therefore conclude that the physiological form of sTREM2 protein functions as efficiently as the sTREM2-171 in modulating microglial responsiveness and decreasing plaque deposition in $5 \times \mathrm{FAD}$ mice.

Long-term expression of sTREM2 reduces A $\beta$ pathology. To validate the findings from recombinant sTREM2 protein and to further assess the long-term impact of sTREM2 on AD-related pathology, we expressed sTREM 2 in the brain of $5 \times$ FAD mice using AAV-mediated expression approach. Viruses carrying cDNAs encoding EGFP alone as a control or EGFP-2A-sTREM2$3 \times$ FLAG (spanning N-terminal amino acids $1-171$ of TREM2) were injected into the cerebral ventricles of P0 neonatal $5 \times \mathrm{FAD}$ mice. Seven months after viral injection, we found similar distribution patterns of EGFP expression in control and sTREM2injected brains, including the cortex and hippocampus (Supplementary Fig. 6a). By analyzing potential colocalization of sTREM2 with cell-type-specific markers for astrocytes, microglia, and neurons, we found that the sTREM2 signal was colocalized primarily with NeuN-positive neurons, but not with GFAPpositive astrocytes or Iba1-positive microglia (Supplementary Fig. 6b). The absence of AAV infection to microglial cells is consistent with the previous observation that microglia are refractory to $\mathrm{AAV}^{49}$. Transduction of primary neurons with the sTREM2 expression construct showed that sTREM2 was efficiently secreted into the media (Supplementary Fig. 6c, d). Thus, sTREM2 possesses the tendency to contact any cell type in the brain, including microglia, after releasing into the extracellular space. The mRNA levels of sTREM2 in the cerebral cortex and hippocampus were significantly increased in $5 \times \mathrm{FAD}$ mice, as compared with the EGFP control (Fig. 6a). This increase was further confirmed at the protein level using ELISA to measure the levels of sTREM2 in the TBS-soluble fractions (Fig. 6b).

We next evaluated the effects of sTREM2 expression on $\mathrm{A} \beta$ levels and plaque deposition. We found that sTREM2 significantly decreased the total plaque burden in both the hippocampus and the cortex of sTREM2-expressing $5 \times \mathrm{FAD}$ mice relative to controls (Fig. $6 \mathrm{c}, \mathrm{d}$ ). Congo red-positive dense core compact plaques were also reduced in the presence of AAVmediated sTREM2 expression (Supplementary Fig. 7a, b). To further analyze how sTREM2 affects the dynamic pools of $A \beta$, we examined $A \beta$ levels in sequentially extracted tris-buffered saline (TBS)-soluble, detergent-soluble (TBSX), and detergent-insoluble (guanidine- $\mathrm{HCl}, \mathrm{GDN}$ ) fractions from cortical and hippocampal mouse brain tissues by ELISA ${ }^{50}$. The majority of $A \beta$ in these mice at 7 months of age was fractioned in the detergentinsoluble fractions, reflecting its deposition in the amyloid plaque (Supplementary Fig. 7c, d). Consistent with decreased A $\beta$ deposition, the concentrations of insoluble $A \beta 42$ in the guanidine fractions were significantly lower in the cortex and hippocampus of sTREM2-expressing $5 \times$ FAD mice than the control. There were no changes in $A \beta$ levels in TBS-soluble and TBSX-soluble fractions. Furthermore, AAV-mediated sTREM2 expression had no significant effects on the levels of APP, its cleavage products, or $A \beta$-degrading enzymes, consistent with the effects that we observed using a recombinant sTREM2 protein (Supplementary Fig. 7e-g). In the presence of sTREM2 expression, the number of plaque-associated microglia was significantly increased in both the hippocampus and the cortex of $5 \times \mathrm{FAD}$ mice (Fig. 6e, f). Taken together, AAV-mediated sTREM2 expression ameliorates plaque deposition and enhances microglial enrichment in the vicinity of an amyloid plaque.

AAV-mediated sTREM2 expression rescues behavioral deficits. We further evaluated if the beneficial effects of sTREM2 expression in reducing amyloid plaque deposition are associated with improved synaptic plasticity and cognitive function of $5 \times \mathrm{FAD}$ mice. To analyze the potential impacts of sTREM2 expression on $\mathrm{AD}$-related behavioral deficits, the spatial learning and memory function of experimental mice were evaluated by the Morris water maze 6 months after viral injection. Similar to the $5 \times$ FAD mice, the mRNA and protein levels of sTREM 2 in the cerebral cortex and the hippocampus detected 7 months after AAV injection were significantly increased in WT mice (Fig. 7a, b). The $5 \times \mathrm{FAD}$ mice were less efficient at finding the hidden platform than WT mice, indicating a deficit in spatial learning ability at 6 months of age (Fig. 7c). Importantly, sTREM2 expression rescued this spatial learning deficit, as evidenced by a reduced latency to reach the platform over the 7 days of training. 
a

\section{PLX3397, $21 d$}

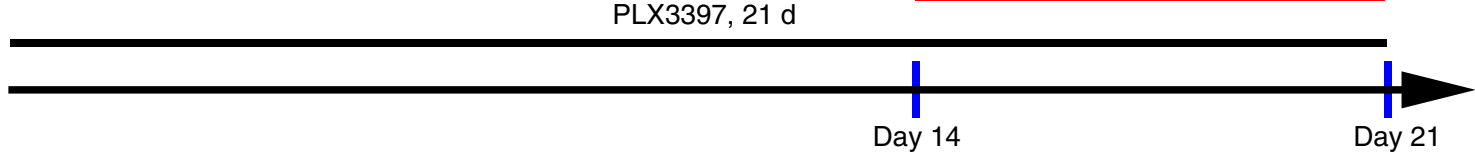

b

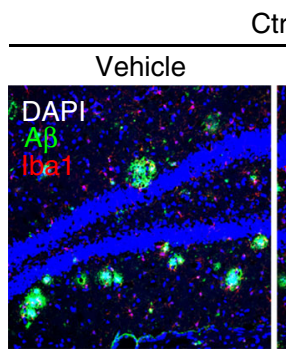

Ctrl

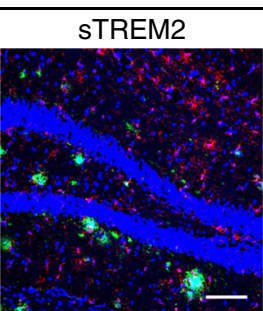

d

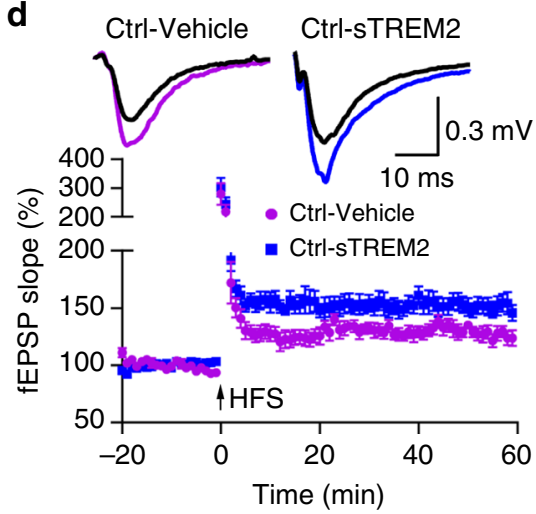

PLX3397
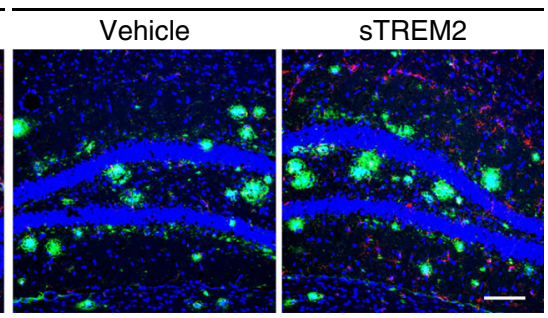

e PLX3397-Vehicle
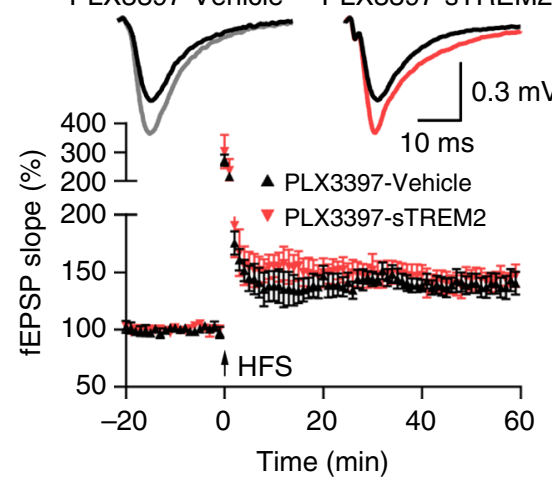

g

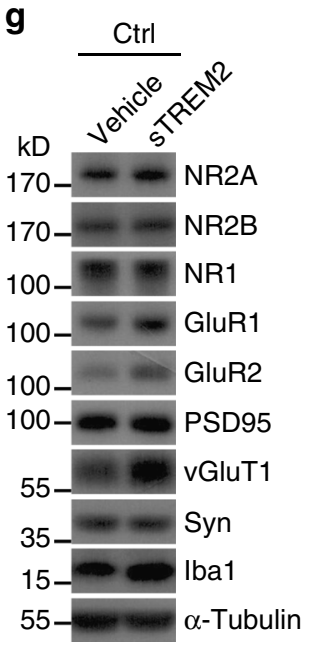

h

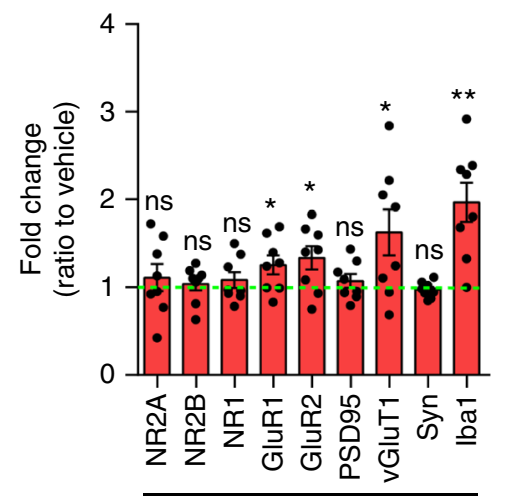

Ctrl
C

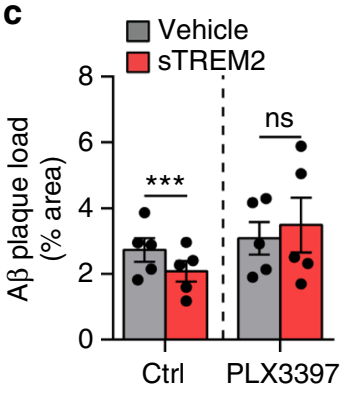

f

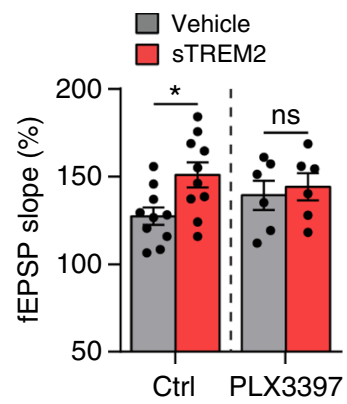

Fig. 4 Depletion of microglia attenuates the protective effects of sTREM2 on A $\beta$ pathology and synaptic plasticity. a Scheme for PLX3397 administration and sTREM2 injection in 5×FAD mice. b The 5×FAD mice were fed with either PLX3397 or control chow for 14 days and then injected with either sTREM2 protein or vehicle control. After continued feeding with PLX3397 or control chow for 7 days, coronal sections were stained with DAPI (blue) for nuclei, MOAB-2 (green) for $A \beta$, and lba1 (red) for microglia. Representative images of the hippocampus region are shown. Original magnification $\times 20$; scale bar, $100 \mu \mathrm{m}$. c Quantitation of amyloid plaque deposition in $\mathbf{b}(n=5$ mice, 18 fields of ctrl and 22 fields of PLX3397 for analysis, paired Student's $t$ test). d, e Brain slices from control chow (d) or PLX3397-fed (e) 5×FAD mice were incubated with $50 \mathrm{nM}$ recombinant sTREM2 protein for $1 \mathrm{~h}$ at room temperature, and transferred to the chamber for LTP recording. Time course of fEPSP measures were recorded in the hippocampal CA1 region before and after $100-\mathrm{Hz}$ stimulation in the Schaffer collateral region. Normalized fEPSP slopes were plotted every 1 min for each group. $\mathbf{f}$ The averaged fEPSPs recorded 50-60 min after induction of LTP ( $n=10$ slices for Ctrl, $n=6$ slices for PLX3397, four mice per group, unpaired Student's $t$ test). $\mathbf{g}$ Synaptic proteins from the hippocampi were analyzed by Western blotting 7 days after vehicle or sTREM2 protein injection to the $5 \times F A D$ mice fed with control chow. $\mathbf{h}$ Quantitation of Western blots in $\mathbf{g}$ ( $n=8$ mice per group, paired Student's $t$ test). i Synaptic proteins from the hippocampi were analyzed by Western blotting 7 days after vehicle or sTREM2 protein injection to the $5 \times$ FAD mice fed with PLX3397. $\mathbf{j}$ Quantitation of Western blots in $\mathbf{i}(n=6$ mice per group, paired Student's $t$ test). All data are presented as mean \pm SEM. ${ }^{\star} p<0.05 ;{ }^{\star \star} p<0.01$; ${ }^{\star \star \star} p<0.001$; ns, not significant 

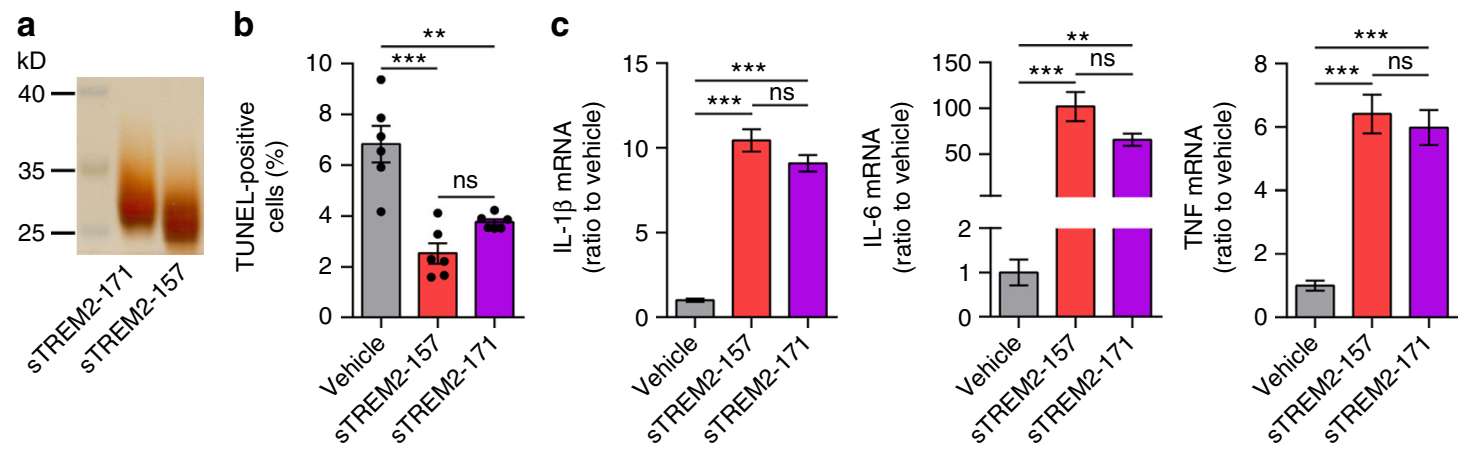

d

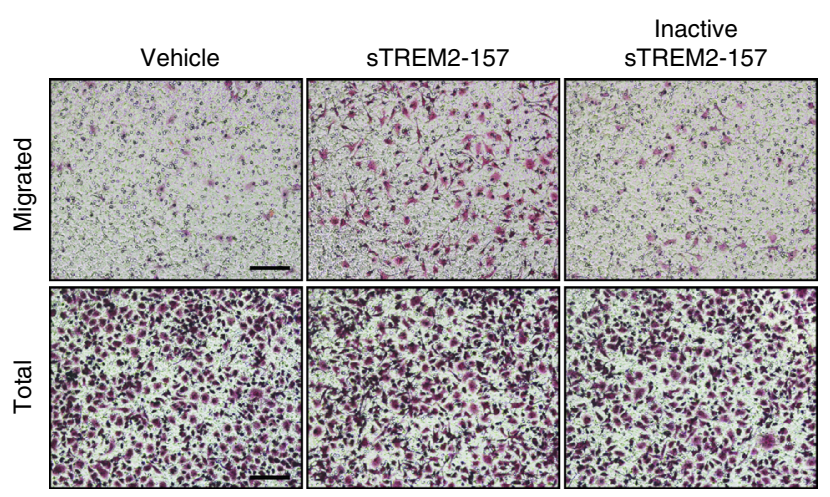

e

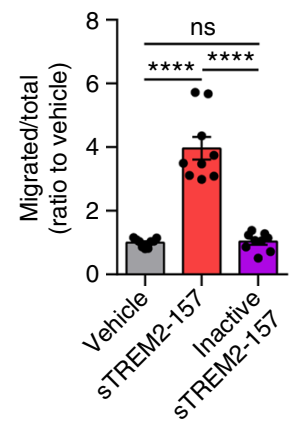

$\mathbf{f}$

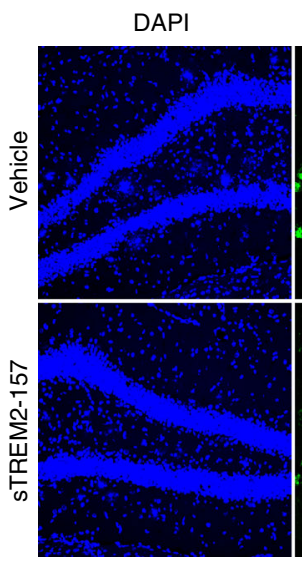

$A \beta$

Iba1
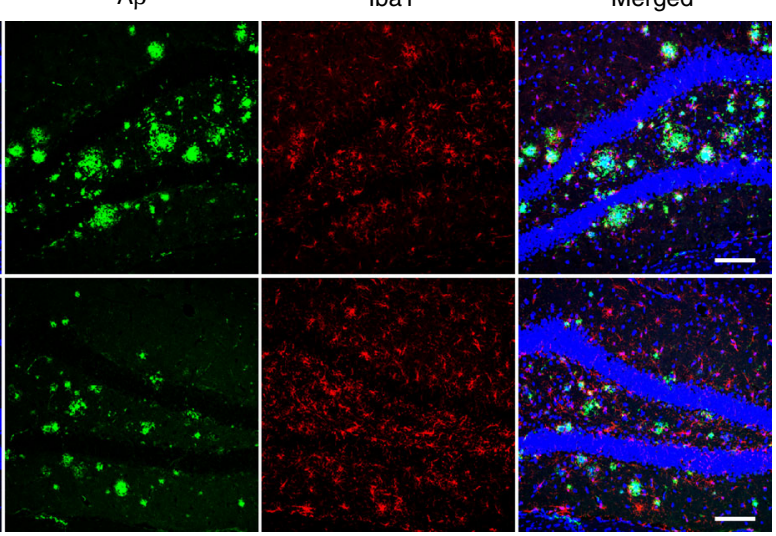

g

$\mathbf{h}$
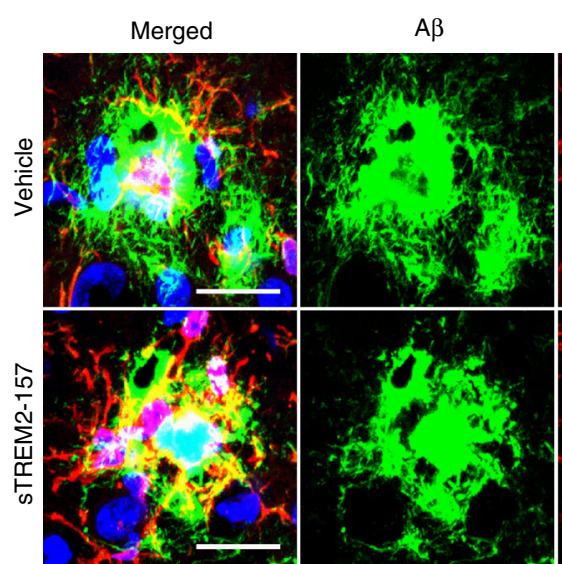

Iba1
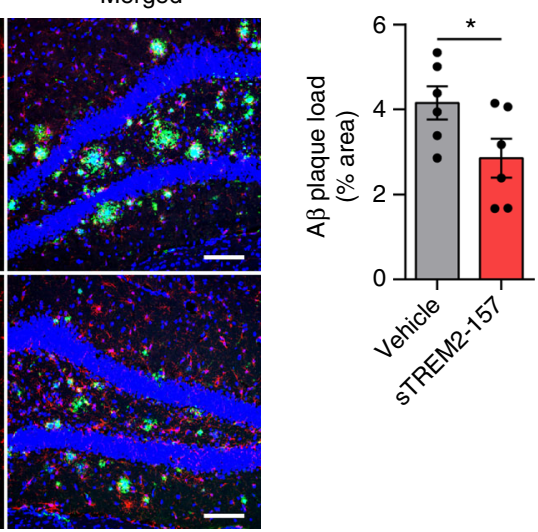

i
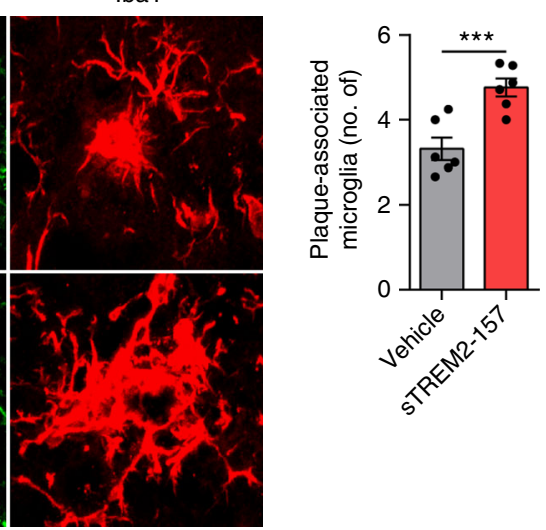

During the probe trials conducted $24 \mathrm{~h}$ after the last training, the $5 \times \mathrm{FAD}$ mice receiving sTREM2 viruses spent significantly more time in the target quadrant than those receiving control viruses (Fig. 7d). However, expression of sTREM2 did not affect the spatial memory of WT mice in either the hidden platform tests or probe trials (Fig. 7c, d). As controls, swim speeds were comparable in all groups of animals, thus excluding impairments in motor function (Fig. 7e). These data indicate that sTREM2 expression could rescue the spatial memory impairments in $5 \times$ FAD mice. 
Fig. 5 The physiological form of STREM2 protein modulates microglial responsiveness and decreases plaque deposition. a The purified sTREM2-157 and sTREM2-171 proteins were analyzed by silver staining. b Primary microglia were cultured with $40 \mathrm{nM}$ sTREM2-157 or sTREM2-171 protein for 24 $\mathrm{h}$ after GM-CSF withdrawal. TUNEL staining was then performed and the number of TUNEL-positive cells were quantified ( $n=6$ from three independent experiments, one-way ANOVA). c Primary microglia were treated with $40 \mathrm{nM}$ sTREM2-157 or sTREM2-171 protein for $4 \mathrm{~h}$. The relative mRNA levels of IL-1 $\beta$, IL- 6 , and TNF shown as bar graphs were determined by quantitative real-time PCR. $\beta$-actin was used as an internal control ( $n=3$ per group, one-way ANOVA). d Primary microglial cells were plated onto transwell chamber inserts. Following 24-h incubation with vehicle (PBS), native sTREM2-157 protein $(100 \mathrm{nM})$, or inactive sTREM2-157 protein $(100 \mathrm{nM})$, cells migrated through the membrane were imaged under a Nikon inverted microscope. Scale bar, $100 \mu \mathrm{m}$. e Quantitation of the number of migrated cells in $\mathbf{d}$ ( $n=9$ from three independent experiments, one-way ANOVA). $\mathbf{f}$ Coronal sections from $5 \times$ FAD mice injected with either sTREM2-157 protein or vehicle control were stained with DAPI (blue) for nuclei, MOAB-2 (green) for A $\beta$, and Iba1 (red) for microglia. Representative images of the hippocampus region are shown. Original magnification $\times 20$; scale bar, $100 \mu \mathrm{m}$. $\mathbf{g}$ Quantitation of amyloid plaque deposition in $\mathbf{f}$ ( $n=6$ mice, 24 fields of each group for analysis, paired Student's $t$ test). $\mathbf{h}$ Coronal sections from sTREM2-157-injected 5×FAD mice were stained with DAPI (blue) for nuclei, MOAB-2 (green) for $A \beta$, and lba1 (red) for microglia. Representative $z$-stack images of the hippocampus region are shown. Scale bar, $25 \mu \mathrm{m}$. i Quantitation of the number of plaque-associated microglia in $\mathbf{h}$ ( $n=6$ mice, 47 plaques of vehicle and 40 plaques of STREM2 for analysis, paired Student's $t$ test). Plaques with $50 \mu \mathrm{m}$ in diameter were selected for analysis. All data are presented as mean $\pm \mathrm{SEM}$. ${ }^{\star} p<0.05$; ${ }^{\star \star} p<0.01 ;{ }^{\star \star \star} p<0.001 ;{ }^{\star \star \star \star} p<0.0001$; ns, not significant

We next examined whether sTREM2-mediated memory improvement in $5 \times \mathrm{FAD}$ mice was reflected at the synaptic level. We thus studied the hippocampal synaptic plasticity in the same set of animals as used for the behavioral study. Consistent with previous studies, the $5 \times \mathrm{FAD}$ mice showed significantly impaired LTP as compared with WT mice (Fig. $7 f, g)^{51}$. Importantly, the magnitude of LTP measured during the last $10 \mathrm{~min}$ post induction (51-60 min) was significantly higher in sTREM2-expressing $5 \times$ FAD mice relative to control mice (Fig. $7 \mathrm{~g}$ ). However, sTREM2 expression had a minimal effect on hippocampal LTP in WT mice. Thus, AAV-mediated expression of sTREM2 largely and specifically ameliorated LTP deficits in $5 \times$ FAD mice. Taken together, our study reveals that sTREM2 expression could rescue or ameliorate key $\mathrm{A} \beta$ /amyloid-induced $\mathrm{AD}$ phenotypes, including defective synaptic plasticity and impaired spatial memory.

\section{Discussion}

Although it has been widely acknowledged that the CSF levels of sTREM2 are elevated in AD, it remains unknown how sTREM2 impacts the amyloid pathology and microglial behaviors in the disease context. In the present study, we demonstrated for the first time that sTREM2 is capable of reducing amyloid deposition, ameliorating synaptic impairments and memory deficits in the $5 \times \mathrm{FAD}$ mouse model. Intriguingly, these protective effects of sTREM2 were attenuated by the depletion of microglia, suggesting a crucial role for microglia in mediating sTREM2 function. Indeed, sTREM2 enhances a variety of microglial activities known to control amyloid pathology, including microgliosis, migration, clustering in the vicinity of an amyloid plaque, as well as uptake and degradation of $A \beta$. As the accumulation and deposition of $A \beta$ in the brain likely drives the pathogenic cascades of $\mathrm{AD}^{4,52,53}$, our study provides a critical link between $\mathrm{A} \beta$ pathology and the emerging role of microglia-mediated innate immune response in the pathogenesis of $\mathrm{AD}^{54,55}$.

Recent studies have begun to unravel the specific roles of TREM2 in modulating microglial functions. For example, the deficiency of TREM2 has been shown to decrease the number of plaque-associated microglia, reduce plaque compaction, and axonal dystrophy ${ }^{11,56}$. The TREM2 signaling pathways also play key roles in regulating $A \beta$ uptake by microglial cells; loss of TREM2 results in reduced phagocytosis, while increased TREM2 gene dosage enhances phagocytic activity ${ }^{11,57}$. Intriguingly, the above-mentioned functions as well as the effects on microglial proliferation and migration by full-length TREM2 are well recapitulated by sTREM2. Since the soluble version of another TREM family member TREM1 was found to negatively modulate TREM1 signaling, initially, sTREM2 was postulated to similarly compete with its membrane-bound form to block TREM2 function. Nevertheless, our data suggest that sTREM2 possesses important biological and pathological roles other than acting as a decoy receptor opposing full-length TREM2 signaling.

Although many functional aspects of sTREM2 resemble those of membrane-bound TREM2, they are not identical. The biological actions of sTREM2 do not require the presence of full-length TREM2 or its intracellular adapter DAP12 ${ }^{43}$. Moreover, the fulllength TREM2 has been found to promote the formation of a neuroprotective microglia barrier that regulates amyloid compaction and insulation, thereby reducing axonal dystrophy around amyloid deposits. Despite a clear reduction of plaque deposition and the total area of dystrophic neurites by sTREM2, it does not significantly impact the amount of dystrophic neurites per plaque and thus unlikely contributes to compacting amyloid fibrils (Fig. 1). Recently, comprehensive single-cell RNA analysis of CNS immune cells in neurodegenerative conditions has discovered an essential role of TREM2 in the activation of disease-associated microglia ${ }^{58,59}$. In future studies, it will be informative to perform transcriptomics analysis at the single-cell level to examine how sTREM2 modulates microglial gene expression in the diseased brain.

A recent study reported that sTREM2 released from cell membranes latches onto amyloid plaque and nearby neurons ${ }^{42}$, indicating a direct physical interaction between sTREM2 and amyloid plaques/neurons. In fact, we have found that an oligomeric A 342 specifically binds to sTREM 2 with a high affinity ${ }^{26,27}$. In addition, sTREM2 also binds to apoE, another major component of amyloid plaques ${ }^{23-25}$. Hence, sTREM2 is likely present in amyloid plaques and acts as a chemoattractant for microglial recruitment to the vicinity of a plaque. Although sTREM2 has been reported to co-localize with neurons in vivo, incubation of primary neuronal cultures with sTREM2 in the absence of microglia had minimal effects on the abundance of the synaptic proteins or the amplitude and frequency of both mEPSCs and mIPSCs (Supplementary Fig. 4). However, sTREM2 significantly increased the levels of several synaptic proteins and rescued the impaired LTP when administered to the brains of $5 \times \mathrm{FAD}$ mice (Fig. 4). Intriguingly, the protective effects of sTREM2 on amyloid load and synaptic function were attenuated by the presence of a selective CSF1R kinase inhibitor, PLX3397, which nearly eliminates microglia from the $5 \times \mathrm{FAD}$ mice (Fig. 4). Hence, our data support that microglia are the main effector cells that mediate the protective functions of sTREM2 in the context of AD. However, the microglial surface receptor(s) for sTREM2 remain unknown. Considering the fact that TREM2 is a highly promiscuous receptor engaging a wide array of ligands, the receptor(s) for sTREM2 might also be highly redundant and needs to be systematically investigated in future studies. 
a

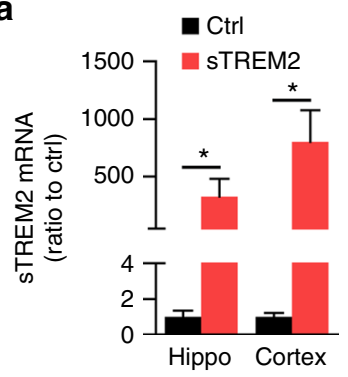

b

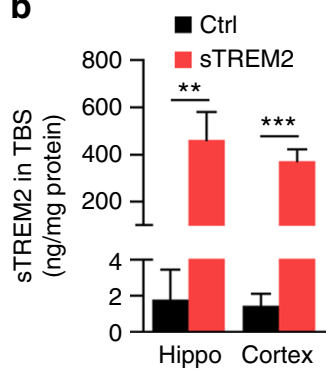

d $\square$ Ctrl

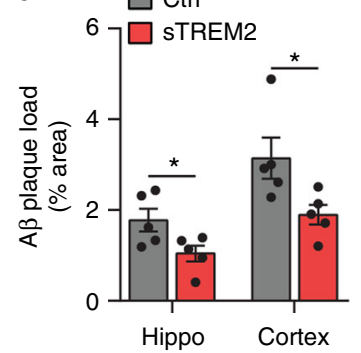

C
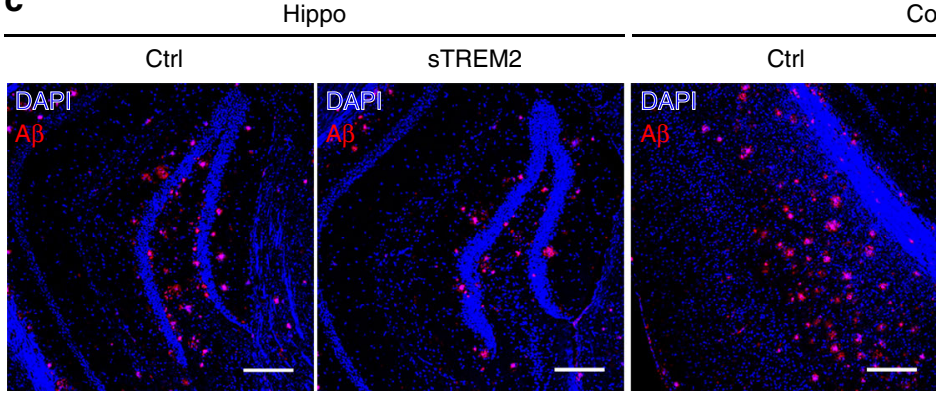

Cortex

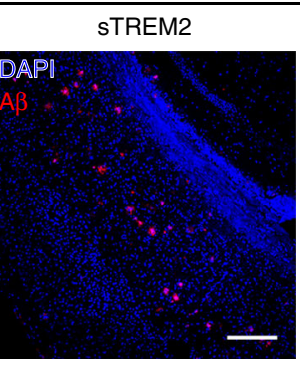

e

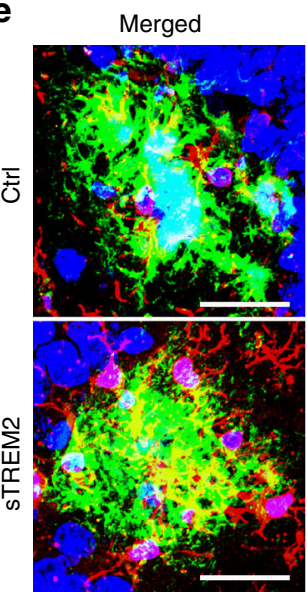

$A \beta$

lba1
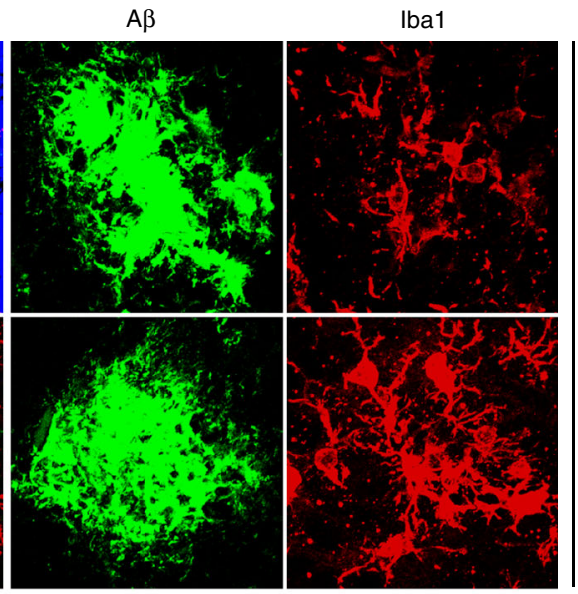

f
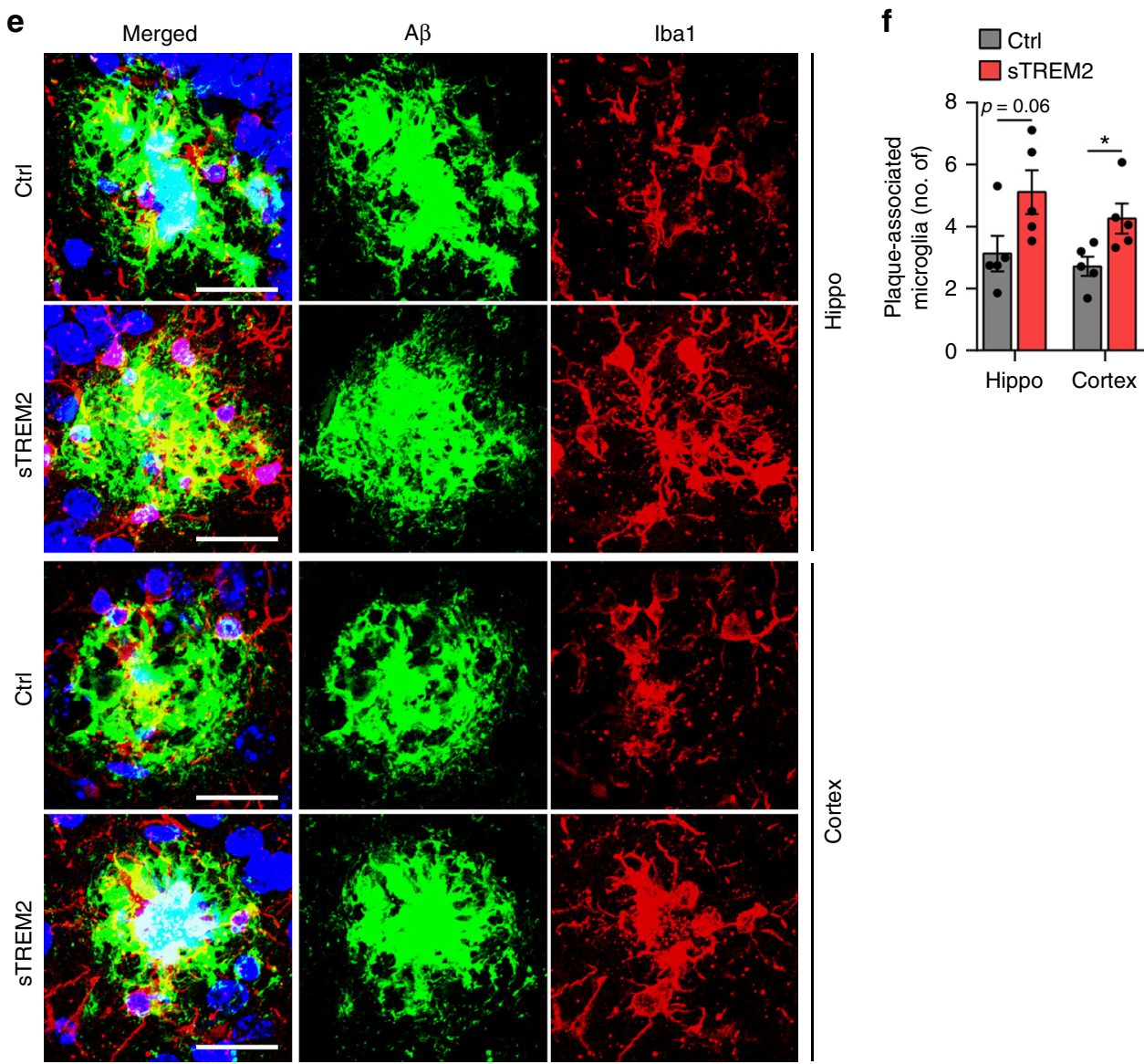

Although the precise mechanism mediating the ability of sTREM2 to attenuate AD pathology remains to be determined, we speculate that significantly reduced levels of $A \beta$ and plaque load upon sTREM2 treatment may contribute to the rescue of synaptic failure and memory deficits in the case of long-term sTREM2 exposure (AAV-mediated sTREM2 expression). However, it is unlikely that the decreased amyloid plaque is responsible for improved LTP in brain slices that were treated with sTREM2 for only $1 \mathrm{~h}$. There are several previous studies that have linked microglia-related functions to synaptic plasticity. For example, Parkhurst et al. showed that microglia promote learning-induced formation of glutamatergic synapses and that this effect is mediated by microglia-released brain-derived neurotrophic factor ${ }^{60}$. In addition, it has been demonstrated that TNF and IL- $1 \beta$ released by microglial cells enhance synaptic transmission and neuronal activity ${ }^{61-63}$. Our current study has clearly shown that sTREM2 exerts its effects in a microgliadependent manner. Thus, it is possible that sTREM2 positively 
Fig. 6 AAV-mediated sTREM2 expression reduces amyloid plaque deposition and increases the number of plaque-associated microglia. a, b AAVmediated sTREM2 expression in 5×FAD mice. Neonatal 5×FAD mice were injected with either AAV2/8-CAG-EGFP (Ctrl) or AAV2/8-CAG-EGFP-2AsTREM2-3×FLAG (human TREM2 1-171 aa) (sTREM2) in the cerebral ventricles and analyzed after 7 months for sTREM2 expression at the mRNA (a, $n=$ 8 mice per group, unpaired Student's $t$ test) or protein ( $\mathbf{b}, n=8$ mice for $C t r l, n=9$ mice for sTREM2, unpaired Student's $t$ test) levels in the cortex and hippcampus using RT-qPCR or ELISA, respectively. c The 5×FAD mice injected with control AAV or AAV-sTREM2 were analyzed for amyloid plaque pathology using an anti-A $\beta$ antibody MOAB-2. Representative images of the hippocampus and the cortex of those mice are shown. Original magnification $\times 10$; scale bar, $200 \mu \mathrm{m}$. Blue, DAPI; red, A $\beta$ antibody MOAB-2. d Quantitation of A $\beta$ plaque area in the hippocampus and cortex of $5 \times F A D$ mice injected with either control AAV or AAV-sTREM2 ( $n=5$ mice per group for the hippocampus or the cortex, 12 fields of the hippo and 28 fields of the cortex for analysis, unpaired Student's $t$ test). e Coronal sections from the 5×FAD mice injected with control AAV or AAV-sTREM2 were stained with DAPI (blue) for nuclei, MOAB-2 (green) for an amyloid plaque, and lba1 (red) for microglia. Representative z-stack images of the hippocampus and cortex region are shown. Scale bar, $25 \mu \mathrm{m}$. f Quantitation of the number of plaque-associated microglia in e $(n=5$ mice, 35 plaques of ctrl and 37 plaques of sTREM2 in the hippocampus or 64 plaques of ctrl and 67 plaques of sTREM2 in the cortex for analysis, unpaired Student's $t$ test). Plaques with $50 \mu m$ in diameter were selected for analysis. All data are presented as mean \pm SEM. ${ }^{\star} p<0.05 ;{ }^{\star \star} p<0.01 ;{ }^{\star \star \star} p<0.001$
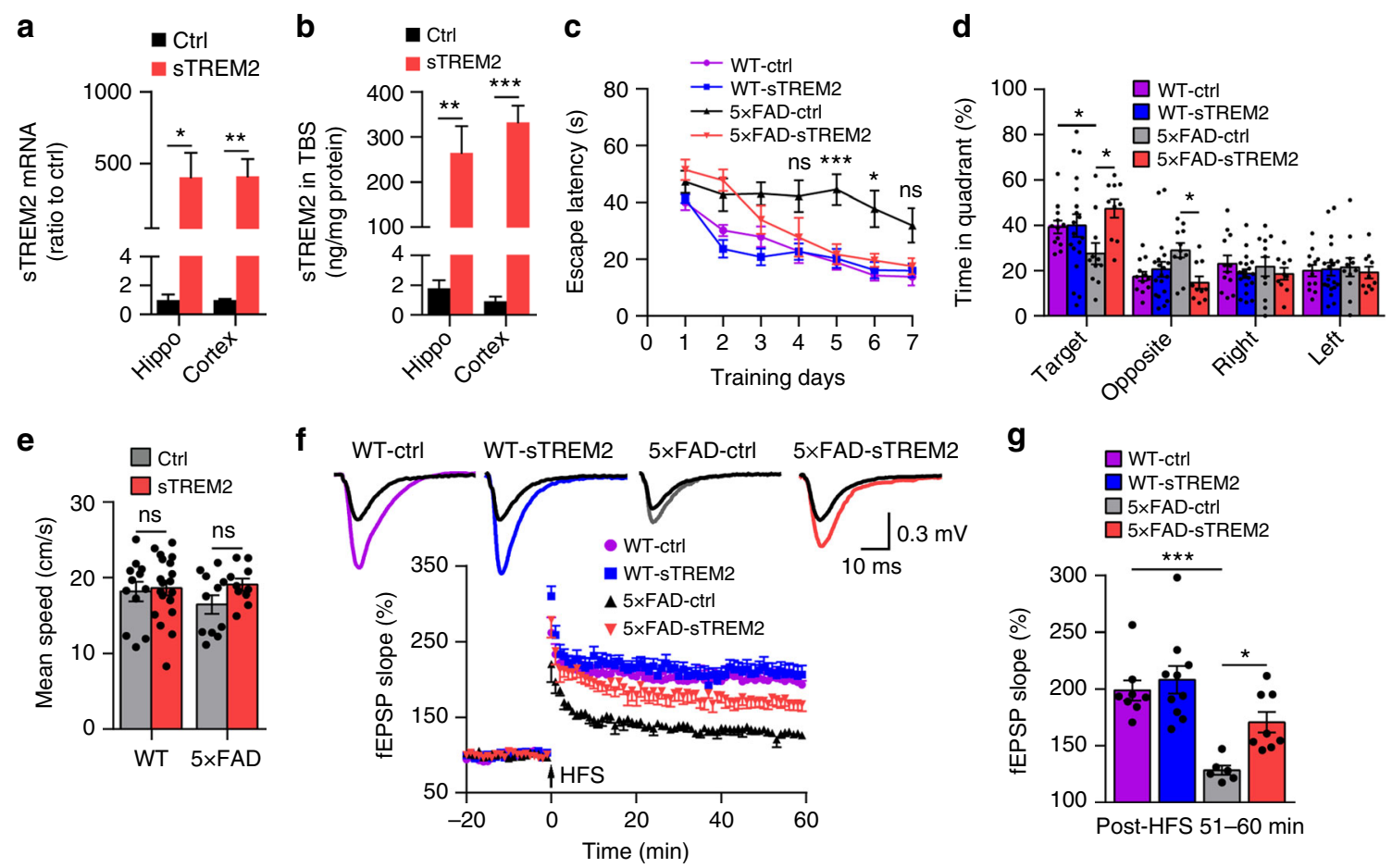

Fig. 7 AAV-mediated sTREM2 expression rescues behavioral and LTP deficits in 5×FAD mice. a, b Neonatal WT mice were injected with either control AAV or AAV-sTREM2 in the cerebral ventricles and analyzed after 7 months for sTREM2 expression at the mRNA (a, $n=6$ mice for Ctrl in hippo or cortex, $n=6$ mice for sTREM2 in hippo and $n=5$ mice for sTREM2 in the cortex, unpaired Student's $t$ test) or protein (b, $n=6$ mice per group, unpaired Student's $t$ test) levels in the cortex and hippcampus using RT-qPCR or ELISA, respectively. c-e Morris water maze tests were performed in wild-type (WT) or $5 \times$ FAD mice 6 months after injection of either control AAV or AAV-sTREM2 ( $n=12$ mice for WT-Ctrl, $n=19$ mice for WT-sTREM2, $n=11$ mice for $5 \times$ FAD-Ctrl, and $n=10$ mice for 5×FAD-sTREM2, two-way ANOVA, Bonferonni post hoc analyses). The escape latency time to reach the hidden platform was recorded during the 7-day training (c). Probe trial was performed $24 \mathrm{~h}$ after the last trial of a hidden platform task and the percentage of search time for each quadrant was recorded (d). Swimming speed of each group was recorded during the probe trial (e). $\mathbf{f}$ Time course of fEPSP measures recorded in the hippocampal CA1 region before and after $100-\mathrm{Hz}$ stimulation in the Schaffer collateral region in slices from WT or $5 \times F A D$ mice 7 months after receiving control AAV or AAV-sTREM2. Normalized fEPSP slopes were plotted every $1 \mathrm{~min}$ for each group. $\mathbf{g}$ The averaged fEPSPs recorded 50-60 min after induction of LTP ( $n=8$ slices for WT-Ctrl, $n=10$ slices for WT-sTREM2, $n=6$ slices for $5 \times$ FAD-Ctrl, and $n=8$ slices for $5 \times$ FAD-sTREM2, at least three mice per group, two-way ANOVA, Bonferonni post hoc analyses). All data are presented as mean $\pm \mathrm{SEM}$. ${ }^{\star} p<0.05 ;{ }^{\star \star} p<0.01$; ${ }^{\star \star \star} p<0.001 ; \mathrm{ns}$, not significant

modulates synaptic plasticity by promoting neurotrophic functions of microglia. Taken together, our data suggest that sTREM2 might exert its protective functions in $\mathrm{AD}$ by both amyloiddependent and amyloid-independent pathways.

In conclusion, our data provide strong evidence that sTREM2 is crucially involved in regulating microglial dynamics to control amyloid plaque development and synaptic plasticity. Although the precise mechanistic underpinnings of the diseasemodulating effects require further investigation, our findings shed new light on the roles of sTREM2 in $\mathrm{AD}$, and suggest that elevation of sTREM2 represents a protective response against $\mathrm{AD}$ pathology.

\section{Methods}

Mice. All animal experiments were approved by the Animal Ethics Committee of the Xiamen University and were conducted in compliance with all relevant ethical regulations for animal testing and research. Sample sizes were adequately powered to observe the effects on the basis of past experience of animal studies ${ }^{43,64}$. Mice 
were randomly selected for further biological analysis, and the investigators were blinded to group allocation during the experiments or outcome assessments.

For AAV injection, postnatal P0 mice within each genotype (WT or $5 \times \mathrm{FAD}$ mice) were injected with control AAV or AAV-sTREM2, and both males and females were used in this study. The behavioral tests were performed at 6 months of age. For LTP and biochemical analysis, samples were harvested from AAVinjected WT or $5 \times \mathrm{FAD}$ mice at 7 months.

For sTREM2 protein injection, $5 \times \mathrm{FAD}$ mice at 7 months of age were injected with control (PBS or heat-inactivated sTREM2) or native sTREM2 protein into the left and right hippocampi, respectively. Seven days after injection, mice were anesthetized and perfused with ice-cold PBS. Samples were harvested for biochemical or histological analysis. Both males and females were used in this study.

To pharmacologically ablate microglia in the brain, 6-month-old (for LTP) or 7-month-old (for amyloid plaque) 5×FAD mice were fed with PLX3397 (Selleck, S7818, $290 \mathrm{mg} / \mathrm{kg}$ formulated in standard chow) ${ }^{47,48}$. Age-matched control groups were fed with the same standard chow but without PLX3397.

Injection of AAV. The AAV2/8 viruses expressing human EGFP-2A-sTREM2$3 \times$ FLAG (1-171aa) (AAV-sTREM2) or EGFP (control AAV) under the control of the CAG promoter were generated by Obio Technology (Shanghai). Neonatal mice were cryoanesthetized on ice for $3 \mathrm{~min}$ before AAV injection. Following cessation of movement, viruses were injected into the lateral ventricles of both cerebral hemispheres with $7.5 \times 10^{9}$ total viral particles per side using a $10-\mu \mathrm{L}$ syringe (Hamilton, 7642-01) with a 30-G needle (Hamilton, 7803-07). After both injections were complete, pups were placed on a warming pad until they regained normal color and resumed movement. All injected pups were then returned to their mothers for care and further recovery.

Tissue preparation. Mice were anesthetized and transcardially perfused with $50 \mathrm{~mL}$ of ice-cold $1 \times$ PBS. Brains were removed and dissected at the midline. For biochemical analysis, the cortex and hippocampus were dissociated and immediately snap-frozen in liquid nitrogen and stored at $-80^{\circ} \mathrm{C}$ for extraction of protein and RNA. For immunohistochemistry analysis, brains were fixed in $4 \%$ paraformaldehyde (PFA, Sigma-Aldrich, P1468) overnight at $4{ }^{\circ} \mathrm{C}$, followed by transferring to $30 \%$ sucrose at $4{ }^{\circ} \mathrm{C}$ for $48 \mathrm{~h}$, before being embedded for cryostat sectioning.

Western blotting. Samples were homogenized and incubated in RIPA Lysis and Extraction Buffer (Thermo Fisher Scientific, 89900), supplemented with Protease and Phosphatase Inhibitor Cocktail (Thermo Fisher Scientific, 78440). Protein concentrations were determined using the BCA Protein Assay Kit (Thermo Fisher Scientific, 23225) according to the manufacturer's instruction. Equal amounts of total proteins were resolved by sodium dodecyl sulfate-polyacrylamide gel electrophoresis and transferred to PVDF membranes (Millipore, IPVH00010). After blocking, the membranes were blotted by a primary antibody and detected with horseradish peroxidase-conjugated secondary antibody. Proteins were visualized using ECL Western blotting detection reagents (Millipore, WBKLS0500). Immunoreactive bands were quantified using ImageJ software. The following antibodies were used: anti-Ibal (Wako, 016-20001, 1:500), anti-GFAP (Cell Signaling Tech nology, 3670S, 1:1000), anti-NR2A (Millipore, 07-632), anti-NR2B (DB Biosciences, 610416, 1:1000), anti-NR1 (Cell Signaling Technology, 5704S, 1:1000), anti-GluR1 (Millipore, MAB2263, 1:1000), anti-GluR2 (Millipore, AB1768-I, 1:1000), anti-PSD95 (Cell Signaling Technology, 3450S, 1:2000), anti-vGluT1 (Millipore, MAB5502, 1:2000), anti-Synaptophysin (Sigma-Aldrich, S5768, 1:2000), anti-APP C-terminal $\left(366^{65}\right.$, in-house, 1:1000), anti-TREM2 (R\&D Systems, AF1828, 1:500), and anti- $\alpha$-Tubulin (Millipore, MABT205, 1:3000).

RNA isolation and real-time quantitative PCR analysis. Total RNA was isolated from the cortex or the hippocampus using TRIzol reagent (Thermo Fisher Scientific, 15596018). One microgram of RNA was reverse transcribed into the firststrand cDNA using cDNA Synthesis SuperMix (TransGen Biotech, China, AT31402) according to the manufacturer's protocol. Quantitative PCR was performed using the FastStart Universal SYBR Green Master mix (Roche, 04913914001). The real-time PCR was performed on 7500 fast (ABI) or 480 LightCycler (Roche). The primer sequences were as follows: Iba1-Forward: GTCCTTGAAGCGAATGCTG G, Iba1-Reverse: CATTCTCAAGATGGCAGATC; GFAP-Forward: TCCTGGA ACAGCAAAACAAG, GFAP-Reverse: CAGCCTCAGGTTGGTTTCAT; IL-1 $\beta$-Forward: GCAACTGTTCCTGAACTCAACT, IL-1 $\beta$-Reverse: ATCTT TTGGGGTCCGTCAACT; IL-6-Forward: CAATGGCAATTCTGATTGTATG, IL-6-Reverse: AGGACTCTGGCTTTGTCTTTC; TNF-Forward: CCCTCACA CTCAGATCATCTTCT, TNF-Reverse: GCTACGACGTGGGCTACAG; sTREM2Forward: AACTTGTGGCTGCTGTCCTT, sTREM2-Reverse: GGTAGAGACCCG CATCATGG; IDE-Forward: AATCCGGCCATCCAGAGAATA, IDE-Reverse: GGGTCTGACAGTGAACCTATGT; NEP-Forward: CTCTCTGTGCTTGTCTTG CTC, NEP-Reverse: GACGTTGCGTTTCAACCAGC; ECE1-Forward: CAGGTG GTCACAGCTCACTAC, ECE1-Reverse: GGTATCCAGTCAGGACCTTTTCA; MMP2-Forward: CAAGTTCCCCGGCGATGTC, MMP2-Reverse: TTCTGG
TCAAGGTCACCTGTC; MMP9-Forward: GCAGAGGCATACTTGTACC, MMP9-Reverse: TGATGTTATGATGGTCCCACTTG.

Microglial migration in transwell assays. Mixed glial cultures were isolated from WT mice at postnatal day 1-2 and were plated onto poly-l-lysine-coated flasks and grown in DMEM supplemented with $10 \%$ fetal bovine serum (FBS) (Gibco). After 3 days, the medium was changed to that which contained $25 \mathrm{ng} / \mathrm{ml}$ granulocytemacrophage CSF (GM-CSF) and 10\% FBS. Primary microglial cells were harvested by shaking after 10-12 days in culture and once every 3 days thereafter (up to four harvests). Microglial migration assay was performed in transwell cell culture inserts with a $8-\mu \mathrm{m}$ pore (Costar, 3422). Primary microglial cells $\left(10^{5}\right)$ suspended in $100 \mu \mathrm{L}$ of serum-free DMEM were added to the upper chamber of the inserts with $600 \mu \mathrm{L}$ of serum-free DMEM in the bottom chamber. After 1-h incubation at $37^{\circ} \mathrm{C}$ and $5 \% \mathrm{CO}_{2}$, the bottom medium was replaced with DMEM containing PBS (vehicle), $100 \mathrm{nM}$ native sTREM2, or heat-inactivated sTREM2 and the culture continued for $24 \mathrm{~h}$. For analysis of the migrated cell number, cells remaining on the upper surface of the membrane were removed by scraping with a cotton swab and the inserts were washed three times with PBS. Cells were then fixed with 4\% PFA for $20 \mathrm{~min}$, followed by staining with hematoxylin and eosin. The total number of cells on the membrane without scraping was also counted in separate wells and the quantification for the microglial migration was normalized to the total final number of cells.

ELISA analyses. For A $\beta$ ELISA, the cortex or the hippocampus was sequentially extracted with TBS, TBSX, and guanidine- $\mathrm{HCl}(\mathrm{GDN})^{50}$. The levels of $\mathrm{A} \beta 40$ or $\mathrm{A} \beta 42$ were measured using sandwich ELISA techniques with monoclonal antibody (mAb) 2.1.3 (human $\mathrm{A} \beta \mathrm{X}-42$ specific, $20 \mu \mathrm{g} / \mathrm{mL}$, in-house) and $\mathrm{mAb}$ 13.1.1 (human $\mathrm{A} \beta \mathrm{X}-40$ specific, $20 \mu \mathrm{g} / \mathrm{mL}$, in-house) for capture and horseradish peroxidase (HRP)-conjugated mAb Ab5 (human A $\beta 1-16$ specific, $1 \mu \mathrm{g} / \mathrm{mL}$, in-house) for detection $^{64}$. The ELISA was developed using ELISA TMB (Sigma-Aldrich, T8665). Synthetic human A $\beta 40$ (AnaSpec, AS-24236) or A $\beta 42$ peptide (AnaSpec, AS20276) was used to generate the standard curves for each assay. Human or mouse sTREM2 ELISA assay was established in-house. A 96-well plate was coated with a TREM2 antibody (R\&D Systems, MAB17291-100, 1:1000) in coating buffer $(0.05$ M Carbonate buffer, $\mathrm{pH}$ 9.6) overnight at $4^{\circ} \mathrm{C}$. The plate was then blocked in blocking buffer (1\% Block Ace in PBS) for $4 \mathrm{~h}$ at room temperature (RT), subsequently washed three times with PBS, and incubated with samples diluted 1:5 with assay buffer ( $0.2 \%$ bovine serum albumin and $0.05 \%$ Tween 20 in PBS) overnight at $4{ }^{\circ} \mathrm{C}$. Plates were washed five times with wash buffer $(0.05 \%$ Tween 20 in PBS) before incubation for $2 \mathrm{~h}$ at RT with human TREM2 biotinylated antibody (R\&D Systems, BAF1828, 1:3000) or mouse Trem2 biotinylated antibody (R\&D Systems, BAF1729, 1:3000). After the washing steps, the plates were incubated with Streptavidin Poly-HRP40 Conjugate (Fitzgerald, 65R-S104PHRP, 1:3000) for $1 \mathrm{~h}$ in the dark. After five additional washing steps, the plates were developed by adding the TMB substrate (Sigma-Aldrich, T5569) and read at $620 \mathrm{~nm}$ on a Varioskan Flash Multimode Reader (Thermo Fisher Scientific).

Immunohistochemistry and microscopy. Mouse coronal sections were cut (12- $\mu \mathrm{m}$ thick) with a cryostat and mounted on poly-L-lysine precoated glass slides, air-dried overnight at $37^{\circ} \mathrm{C}$, and subsequently processed for staining. The sections were washed in $1 \times$ PBS for 15 min and incubated with blocking buffer (PBS with $5 \%$ normal donkey serum and $0.2 \%$ Triton X-100) for $1 \mathrm{~h}$ at RT, followed by incubation with primary antibodies for $48 \mathrm{~h}$ at $4{ }^{\circ} \mathrm{C}$ and the Alexa-fluorophoreconjugated secondary antibodies (Thermo Fisher Scientific, A-21203, A-21202, A21207, A-21244, or A-21247, 1:400) for $2 \mathrm{~h}$ at RT in the dark. Sections were washed and sealed with an anti-fade reagent (Life Technologies, P36935). For confocal microscopy, a NIKON A1R Plus confocal microscope was used to acquire all images; laser and detector settings were maintained constant in the same experiment. For analysis of the amyloid plaque-associated microglia, $6-\mu \mathrm{m}$ z-stacks (consisting of 13 optical slices of $0.5-\mu \mathrm{m}$ thickness) were acquired and the maximum intensity projections were generated. To examine the colocalization of an amyloid plaque and CD68, 6- $\mu \mathrm{m}$ z-stacks (consisting of 13 optical slices of $0.5-\mu \mathrm{m}$ thickness) were acquired and 3D reconstruction was conducted using the Imaris software (Bitplane). The following antibodies of immunohistochemistry detection were used in this study: anti-Ibal (Wako, 019-19741, 1:200), anti-A $\beta$ (MOAB-2, Abcam, ab126649, 1:400), anti-TREM2 (R\&D Systems, AF1828, 1:50), anti-Lamp1 (DB Biosciences, 553792, 1:50), anti-Ki67 (ThermoFisher, 14-5698-82, 1:50), antiCD68 (Bio-Rad, MCA1957, 1:50), anti-NeuN (R\&D Systems, MAB377, 1:100), and anti-GFAP (R\&D Systems, AB5804, 1:800).

Brain stereotaxic injection. The $5 \times \mathrm{FAD}$ mice at 7 months of age were anesthetized and placed in a stereotaxic frame, a skin incision was made, and holes were drilled at $\mathrm{x}( \pm 2.0 \mathrm{~mm}$ from bregma) and y $(-2.0 \mathrm{~mm}$ from bregma). A total of $6 \mu \mathrm{g}$ of sTREM2 protein in $2.0 \mu \mathrm{L}$ of PBS, or heat-inactivated sTREM2 in $2.0 \mu \mathrm{L}$ of PBS, or $2.0 \mu \mathrm{L}$ of PBS alone was delivered at $0.20 \mu \mathrm{L} / \mathrm{min}$ at z-depths of $2.0 \mathrm{~mm}$ to the right and left hemispheres, respectively. The syringe was left in place for $10 \mathrm{~min}$ after each injection before being withdrawn slowly. Seven days after injection, mice were anesthetized and perfused with ice-cold PBS. For biochemical analysis, hippocampi from the left or the right brain were dissected out, snap-frozen in liquid 
nitrogen, and stored at $-80^{\circ} \mathrm{C}$ for extraction of protein and RNA. For histologic analysis, brains were fixed in $4 \%$ PFA overnight at $4{ }^{\circ} \mathrm{C}$ and transferred to $30 \%$ sucrose for $48 \mathrm{~h}$ before being embedded for cryostat sectioning.

A $\beta$ phagocytosis assays. For the in vitro assay, primary microglial cells were seeded into 12 -well culture plates at a density of $2 \times 10^{5}$ cells per well and were cultured for $24 \mathrm{~h}$ after GM-CSF withdrawal. Cells were treated with $160 \mathrm{nM}$ sTREM2 protein in serum-free DMEM for $12 \mathrm{~h}$, followed by treatment with a phagocytosis inhibitor cytochalasin D (Calbiochem, 250255, $10 \mu \mathrm{M}$ ) for $30 \mathrm{~min}$ and incubation with $500 \mathrm{nM}$ FAM-A $\beta 42$ oligomer for an additional $3 \mathrm{~h}$. Cells were trypsinized and washed with cold PBS for detection of FAM fluorescence using fluorescence-activated cell sorting (FACS). The in vivo phagocytosis assay was performed, as described previously with slight modifications ${ }^{66}$. The 7-month-old $5 \times \mathrm{FAD}$ mice were injected with sTREM2 as described above. After 7 days, mice were injected intraperitoneally with methoxy-X04 (Toris, 4920) at $10 \mathrm{mg} / \mathrm{kg}$ in $10 \%$ DMSO/90\% PBS ( $\mathrm{pH} 12$ ) for $3 \mathrm{~h}$. Anesthetized mice were perfused with ice-cold PBS and the hippocampi were isolated and chopped into pieces and digested in DMEM/F12 containing $1 \mathrm{mg} / \mathrm{mL}$ Papain (Sigma, P4762), $1.2 \mathrm{U} / \mathrm{mL}$ Dispase II (Sigma, D4693), $20 \mathrm{U} / \mathrm{mL}$ DNAse I (Sigma, D5025), and $100 \mathrm{U} / \mathrm{mL}$ Collagenase IV (ThermoFisher, 17104019) at $37^{\circ} \mathrm{C}$ for $30 \mathrm{~min}$. The homogenization was achieved by pipetting gently up and down. The homogenates were washed and filtered through a $70-\mu \mathrm{m}$ cell strainer. Hippocampal cells were centrifuged at $500 \mathrm{~g}$ for 5 min at $4{ }^{\circ} \mathrm{C}$ and resuspended in PBS. Cells were incubated with the $\mathrm{Fc}$ receptor blocking antibody CD16/CD32 (BD Bioscience, 553141, 1:100) for $10 \mathrm{~min}$ at $4^{\circ} \mathrm{C}$ to prevent unspecific binding. Cells were washed once and then resuspended in $200 \mu \mathrm{L}$ of PBS containing CD11b-APC (eBioscience, 17-0112-82, 1:100) and CD45FITC (eBioscience, 11-0451-85, 1:100) and incubated for $1 \mathrm{~h}$ at $4{ }^{\circ} \mathrm{C}$. Cells were washed again and resuspended in $2 \%$ FBS/PBS for FACS analysis. The frequencies of viable methoxy-X04 ${ }^{+} \mathrm{CD} 11 \mathrm{~b}^{+} \mathrm{CD} 45^{\text {low }}$ microglia were measured by flow cytometry using a CytoFlex S (Beckman) and analyzed using FlowJo (Tree Star). WT mice injected with methoxy-X04 were used as a control to determine the methoxyX04 threshold for non-phagocytosing cells.

Intracellular $\mathbf{A} \boldsymbol{\beta}$ clearance. Primary microglia were treated with vehicle or sTREM2 protein $(160 \mathrm{nM})$ in serum-free DMEM medium for $12 \mathrm{~h}$, followed by treatment with lysosomal enzyme inhibitors (Pepstatin A: $10 \mu \mathrm{M}$, Leupeptin: $100 \mu \mathrm{M}$, and E-64d: $50 \mu \mathrm{M}$ ) or chloroquine (Sigma-Aldrich, C6628, $40 \mu \mathrm{M}$ ) for $30 \mathrm{~min}$ and incubation with a $500 \mathrm{nM}$ A $\beta 42$ oligomer for an additional $3 \mathrm{~h}$. Cells were harvested with trypsin and cell pellets were washed twice with PBS. Microglia were lysed in lysis buffer ( $5 \mathrm{M}$ guanidine in $50 \mathrm{mM}$ Tris- $\mathrm{HCl}, \mathrm{pH}$ 8.0). Lysates were centrifuged at $13,400 \mathrm{~g}$ at $4{ }^{\circ} \mathrm{C}$ for $15 \mathrm{~min}$. Supernatants were collected and further used for quantifying the amounts of cell-associated $A \beta 42$ using ELISA. The amounts of degraded $A \beta 42$ were calculated by subtracting the cell-associated $A \beta$ in the absence of inhibitors from that in the presence of inhibitors.

Electrophysiological recording. For LTP recording, experiments were performed, as previously reported with modifications ${ }^{67}$. In brief, animals were deeply anesthetized with isoflurane and killed by decapitation. The brain was quickly removed and dissected in an ice-cold cutting solution containing $64 \mathrm{mM} \mathrm{NaCl}, 2.5 \mathrm{mM} \mathrm{KCl}$, $1.25 \mathrm{mM} \mathrm{NaH}_{2} \mathrm{PO}_{4}, 10 \mathrm{mM} \mathrm{MgSO}_{4}, 0.5 \mathrm{mM} \mathrm{CaCl}_{2}, 26 \mathrm{mM} \mathrm{NaHCO}_{3}, 10 \mathrm{mM}$ glucose, and $120 \mathrm{mM}$ sucrose. Acute coronal slices $(400 \mu \mathrm{m})$ were prepared using a Vibroslice (VT $1000 \mathrm{~S}$; Leica). Slices were allowed to recover for $30 \mathrm{~min}$ at $34^{\circ} \mathrm{C}$ and then at RT for at least $1 \mathrm{~h}$ before recording in the artificial CSF (aCSF) containing $126 \mathrm{mM} \mathrm{NaCl}, 2.5 \mathrm{mM} \mathrm{KCl}, 1.25 \mathrm{mM} \mathrm{NaH}_{2} \mathrm{PO}_{4}, 2 \mathrm{mM} \mathrm{MgSO}_{4}, 2 \mathrm{mM}$ $\mathrm{CaCl}_{2}, 26 \mathrm{mM} \mathrm{NaHCO}_{3}$, and $10 \mathrm{mM}$ glucose. Slices were transferred to the recording chamber and superfused with aCSF $(2 \mathrm{~mL} / \mathrm{min})$ saturated with $95 \% \mathrm{O}_{2} /$ $5 \% \mathrm{CO}_{2}$ (volume/volume) at $34^{\circ} \mathrm{C}$. For sTREM2 protein treatment, brain slices were incubated with $50 \mathrm{nM}$ recombinant sTREM2 protein for $1 \mathrm{~h}$ at RT, and transferred to the chamber for LTP recording. fEPSPs were evoked every $20 \mathrm{~s}$ in the CA1 stratum radiatum by stimulating the SCs/commissural pathway (for CA1) with a bipolar stimulating electrode (FHC, Inc.) and recorded using a Multi-Clamp 700B amplifier (Molecular Devices) and Clampex10.5 acquisition software (Molecular Devices) and digitized with Digidata 1550 A (Molecular Devices) with glass pipettes (1-3 M $\Omega$ ) filled with aCSF. Test stimuli consisted of monophasic 0.1ms pulses of constant currents (with intensity adjusted to produce $25 \%$ of the maximum response) at a frequency of $0.05 \mathrm{~Hz}$. After a 20 -min-stable baseline was established, LTP was induced in the CA1 area by two trains of $100-\mathrm{Hz}$ stimuli with the same intensity of the test stimulus. The strength of synaptic transmission was determined by measuring the initial (20-80\% rising phase) slope of fEPSPs.

Whole-cell voltage-clamp recordings were performed on cortical neurons cultured for 12-14 DIV ${ }^{68}$. For sTREM2 protein treatment, neurons were incubated with $50 \mathrm{nM}$ recombinant sTREM2-Fc or Fc protein prepared as previously described ${ }^{43}$ for $1 \mathrm{~h}$, and transferred to the chamber for analysis. Recordings were conducted in a submerged recording chamber perfused $(1-1.5 \mathrm{~mL} / \mathrm{min})$ with aCSF containing (in mM) $126 \mathrm{NaCl}, 2.5 \mathrm{KCl}, 1.2 \mathrm{NaH}_{2} \mathrm{PO}_{4}, 2.4 \mathrm{MgCl} \cdot 6 \mathrm{H}_{2} \mathrm{O}, 1.2 \mathrm{CaCl}_{2}$, $18 \mathrm{NaHCO}_{3}$, and 11 Glucose, equilibrated with $95 \% \mathrm{O}_{2}$ and $5 \% \mathrm{CO}_{2}$ at RT. The patch pipette (5-8 $\mathrm{M} \Omega$ ) was filled with a standard intracellular solution containing (in mM) $140 \mathrm{CsCH}_{3} \mathrm{SO}_{3}, 2 \mathrm{MgCl} \cdot 6 \mathrm{H}_{2} \mathrm{O}, 5 \mathrm{TEA}-\mathrm{Cl}, 10$ HEPES, $2.5 \mathrm{MgATP}$, $0.3 \mathrm{Na}_{2} \mathrm{GTP}$, and 1 EGTA, pH 7.2-7.4 (with CsOH). mEPSCs and mIPSCs were recorded with $1 \mu \mathrm{M}$ tetrodotoxin (TTX; Tocris, Cat. No. 1069) in voltage-clamp mode at a holding potential of $-70 \mathrm{mV}$ and $0 \mathrm{mV}$, respectively. Series resistance was monitored throughout the recording and was $<30 \mathrm{M} \Omega$. Data were filtered at $2 \mathrm{kHz}$ and sampled at $10 \mathrm{kHz}$. Whole-cell recording was made using patch-clamp amplifiers (Multiclamp 700B). Data acquisition was performed using digitizers (DigiData 1440A). mEPSC and mIPSC were analyzed off-line with MiniAnalysis software (Synaptosoft Inc., Fort Lee, NJ).

Morris water maze. Experiments were conducted in a circular 120 -cm-diameter pool filled with opaque water kept at $21^{\circ} \mathrm{C}$. The mice were given four training trials per day for 7 consecutive days, during which the platform was left in the same position. The time taken to reach the platform (escape latency) was measured, and the average of four trials was determined. If the mouse could not find the escape platform within $60 \mathrm{~s}$, it was placed on the platform for $10 \mathrm{~s}$. A probe trail without a platform was performed $24 \mathrm{~h}$ after the last trial of the hidden platform test. Percentage search time for each quadrant was recorded for $60 \mathrm{~s}$. Mice were tracked by a video camera (SONY) in both training and probe trials. Collected data were analyzed by SMART 2.5 VIDEO TRACKING software (Panlab, Harvard Apparatus).

Statistical analysis. All the electrophysiological data and histologic data analyses were performed blindly. Statistical tests were performed using GraphPad Prism 5 or 6 (GraphPad Software, La Jolla, CA). Two-way ANOVA followed by Bonferonni post hoc analyses or unpaired Student's $t$ test was used to analyze AAV and neuron data Paired Student's $t$ test was used to analyze sTREM2 protein-injected experiments. The statistical method for each quantitated data was described in the Figure legend. All data are shown as mean \pm SEM and specific $n$ values were reported in each Figure legend. Levels of significance are as follows: ${ }^{*} p<0.05,{ }^{* *} p<0.01,{ }^{* * *} p<0.001,{ }^{* * * *} p<0.0001$. $p<0.05$ was considered as stastically significant.

Reporting Summary. Further information on experimental design is available in the Nature Research Reporting Summary linked to this article.

\section{Data availability}

The data underlying Figs. 1c, 1d, 1f, 1h, 1i, 2a, 2c, 2e, 2f, 2h, 3b, 3d, 3f, 3h, 3i, 3j, 4c, 4d, $4 \mathrm{e}, 4 \mathrm{f}, 4 \mathrm{~h}, 4 \mathrm{j}, 5 \mathrm{~b}, 5 \mathrm{c}, 5 \mathrm{e}, 5 \mathrm{~g}, 5 \mathrm{i}, 6 \mathrm{a}, 6 \mathrm{~b}, 6 \mathrm{~d}, 6 \mathrm{f}, 7 \mathrm{a}, 7 \mathrm{~b}, 7 \mathrm{c}, 7 \mathrm{~d}, 7 \mathrm{e}, 7 \mathrm{f}, 7 \mathrm{~g}$, and Supplementary Figs. 1a, 2a, 2b, 3a, 3b, 3c, 3d, 3e, 4b, 4c, 4d, 4e, 4f, 4g, 4i, 4k, 4l, 4m, 5c, 5e, 6c, 6d, 7b, 7c, $7 \mathrm{~d}, 7 \mathrm{e}, 7 \mathrm{f}, 7 \mathrm{~g}$ are provided as a Source Data file. All other data that support the findings of this study are available from the corresponding author upon request.

Received: 7 September 2018 Accepted: 19 February 2019

Published online: 25 March 2019

\section{References}

1. Heneka, M. T. et al. Neuroinflammation in Alzheimer's disease. Lancet Neurol. 14, 388-405 (2015).

2. Serrano-Pozo, A., Frosch, M. P., Masliah, E. \& Hyman, B. T. Neuropathological alterations in Alzheimer disease. Cold Spring Harb. Perspect. Med. 1, a006189 (2011).

3. Holtzman, D. M., Morris, J. C. \& Goate, A. M. Alzheimer's disease: the challenge of the second century. Sci. Transl. Med. 3, 77sr71 (2011).

4. Hardy, J. A. \& Higgins, G. A. Alzheimer's disease: the amyloid cascade hypothesis. Science 256, 184-185 (1992).

5. Yoon, S. S. \& Jo, S. A. Mechanisms of amyloid-beta peptide clearance: potential therapeutic targets for Alzheimer's disease. Biomol.Ther. 20, 245-255 (2012).

6. Lee, C. Y. \& Landreth, G. E. The role of microglia in amyloid clearance from the AD brain. J. Neural Transm. 117, 949-960 (2010).

7. McGeer, P. L., Itagaki, S., Tago, H. \& McGeer, E. G. Reactive microglia in patients with senile dementia of the Alzheimer type are positive for the histocompatibility glycoprotein HLA-DR. Neurosci. Lett. 79, 195-200 (1987).

8. Perlmutter, L. S., Barron, E. \& Chui, H. C. Morphologic association between microglia and senile plaque amyloid in Alzheimer's disease. Neurosci. Lett. 119, 32-36 (1990).

9. Stalder, M. et al. Association of microglia with amyloid plaques in brains of APP23 transgenic mice. Am. J. Pathol. 154, 1673-1684 (1999).

10. Condello, C., Yuan, P., Schain, A. \& Grutzendler, J. Microglia constitute a barrier that prevents neurotoxic protofibrillar Abeta42 hotspots around plaques. Nat. Commun. 6, 6176 (2015).

11. Yuan, P. et al. TREM2 haplodeficiency in mice and humans impairs the microglia barrier function leading to decreased amyloid compaction and severe axonal dystrophy. Neuron 90, 724-739 (2016). 
12. Bertram, L. et al. Genome-wide association analysis reveals putative Alzheimer's disease susceptibility loci in addition to APOE. Am. J. Human Genet. 83, 623-632 (2008).

13. Guerreiro, R. et al. TREM2 variants in Alzheimer's disease. New Engl. J. Med. 368, 117-127 (2013)

14. Hollingworth, P. et al. Common variants at ABCA7, MS4A6A/MS4A4E, EPHA1, CD33 and CD2AP are associated with Alzheimer's disease. Nat. Genet. 43, 429-435 (2011).

15. Jin, S. C. et al. Coding variants in TREM2 increase risk for Alzheimer's disease. Human Mol. Genet. 23, 5838-5846 (2014).

16. Jonsson, T. et al. Variant of TREM2 associated with the risk of Alzheimer's disease. New Engl. J. Med. 368, 107-116 (2013).

17. Naj, A. C. et al. Common variants at MS4A4/MS4A6E, CD2AP, CD33 and EPHA1 are associated with late-onset Alzheimer's disease. Nat. Genet. 43, 436-441 (2011).

18. Sims, R. et al. Rare coding variants in PLCG2, ABI3, and TREM2 implicate microglial-mediated innate immunity in Alzheimer's disease. Nat. Genet. 49, 1373-1384 (2017).

19. Efthymiou, A. G. \& Goate, A. M. Late onset Alzheimer's disease genetics implicates microglial pathways in disease risk. Mol. Neurodegener. 12, 43 (2017).

20. Schmid, C. D. et al. Heterogeneous expression of the triggering receptor expressed on myeloid cells- 2 on adult murine microglia. J. Neurochem. 83 , 1309-1320 (2002).

21. Jay, T. R., von Saucken, V. E. \& Landreth, G. E. TREM2 in neurodegenerative diseases. Mol. Neurodegener. 12, 56 (2017).

22. Wang, Y. M. et al. TREM2 lipid sensing sustains the microglial response in an Alzheimer's disease model. Cell 160, 1061-1071 (2015).

23. Atagi, Y. et al. Apolipoprotein $\mathrm{E}$ is a ligand for triggering receptor expressed on myeloid cells 2 (TREM2). J. Biol. Chem. 290, 26043-26050 (2015).

24. Bailey, C. C., DeVaux, L. B. \& Farzan, M. The triggering receptor expressed on myeloid cells 2 binds apolipoprotein E. J. Biol. Chem. 290, 26033-26042 (2015).

25. Yeh, F. L., Wang, Y., Tom, I., Gonzalez, L. C. \& Sheng, M. TREM2 binds to apolipoproteins, including APOE and CLU/APOJ, and thereby facilitates uptake of amyloid-beta by microglia. Neuron 91, 328-340 (2016).

26. Zhao, Y. et al. TREM2 is a receptor for beta-amyloid that mediates microglial function. Neuron 97, 1023-1031 e1027 (2018).

27. Zhong, L. et al. Amyloid-beta modulates microglial responses by binding to the triggering receptor expressed on myeloid cells 2 (TREM2). Mol. Neurodegener. 13, 15 (2018).

28. Gratuze, M., Leyns, C. E. G. \& Holtzman, D. M. New insights into the role of TREM2 in Alzheimer's disease. Mol. Neurodegener. 13, 66 (2018).

29. Filipello, F. et al. The microglial innate immune receptor TREM2 is required for synapse elimination and normal brain connectivity. Immunity 48, 979-991 e978 (2018).

30. Ulland, T. K. et al. TREM 2 maintains microglial metabolic fitness in Alzheimer's disease. Cell 170, 649-663 e613 (2017).

31. Zheng, H. et al. TREM2 promotes microglial survival by activating Wnt/betacatenin pathway. J. Neurosci. 37, 1772-1784 (2017).

32. Zhong, L. et al. DAP12 stabilizes the C-terminal fragment of the triggering receptor expressed on myeloid cells-2 (TREM2) and protects against LPSinduced pro-inflammatory response. J. Biol. Chem. 290, 15866-15877 (2015).

33. Jay, T. R. et al. TREM 2 deficiency eliminates TREM $2+$ inflammatory macrophages and ameliorates pathology in Alzheimer's disease mouse models. J. Exp. Med. 212, 287-295 (2015).

34. Jay, T. R. et al. Disease progression-dependent effects of TREM2 deficiency in a mouse model of Alzheimer's Disease. J. Neurosci. 37, 637-647 (2017).

35. Feuerbach, D. et al. ADAM17 is the main sheddase for the generation of human triggering receptor expressed in myeloid cells (hTREM2) ectodomain and cleaves TREM2 after Histidine 157. Neurosci. Lett. 660, 109-114 (2017).

36. Schlepckow, K. et al. An Alzheimer-associated TREM2 variant occurs at the ADAM cleavage site and affects shedding and phagocytic function. Embo Mol. Med 9, 1356-1365 (2017).

37. Thornton, P. et al. TREM 2 shedding by cleavage at the H157-S158 bond is accelerated for the Alzheimer's disease-associated H157Y variant. Embo Mol. Med 9, 1366-1378 (2017).

38. Suarez-Calvet, M. et al. sTREM2 cerebrospinal fluid levels are a potential biomarker for microglia activity in early-stage Alzheimer's disease and associate with neuronal injury markers. Embo Mol. Med 8, 466-476 (2016).

39. Heslegrave, A. et al. Increased cerebrospinal fluid soluble TREM2 concentration in Alzheimer's disease. Mol. Neurodegener. 11, 3 (2016).

40. Suarez-Calvet, M. et al. Early changes in CSF sTREM2 in dominantly inherited Alzheimer's disease occur after amyloid deposition and neuronal injury. Sci. Transl. Med. 8, 369ra178 (2016).

41. Piccio, L. et al. Cerebrospinal fluid soluble TREM2 is higher in Alzheimer disease and associated with mutation status. Acta Neuropathol. 131, 925-933 (2016).
42. Song, W. M. et al. Humanized TREM2 mice reveal microglia-intrinsic and -extrinsic effects of R47H polymorphism. J. Exp. Med. 215, 745-760 (2018).

43. Zhong, L. et al. Soluble TREM2 induces inflammatory responses and enhances microglial survival. J. Exp. Med. 214, 597-607 (2017).

44. Hansen, D. V., Hanson, J. E. \& Sheng, M. Microglia in Alzheimer's disease. J. Cell Biol. 217, 459-472 (2018).

45. Sasaki, Y., Ohsawa, K., Kanazawa, H., Kohsaka, S. \& Imai, Y. Iba1 is an actincross-linking protein in macrophages/microglia. Biochem. Biophys. Res. Commun. 286, 292-297 (2001).

46. Ahmed, Z. et al. Actin-binding proteins coronin-1a and IBA-1 are effective microglial markers for immunohistochemistry. J. Histochem. Cytochem. 55, 687-700 (2007).

47. Elmore, M. R. et al. Colony-stimulating factor 1 receptor signaling is necessary for microglia viability, unmasking a microglia progenitor cell in the adult brain. Neuron 82, 380-397 (2014).

48. Spangenberg, E. E. et al. Eliminating microglia in Alzheimer's mice prevents neuronal loss without modulating amyloid-beta pathology. Brain 139, 1265-1281 (2016).

49. Rosario, A. M. et al. Microglia-specific targeting by novel capsid-modified AAV6 vectors. Mol. Ther. Methods Clin. Dev. 3, 16026 (2016).

50. Youmans, K. L. et al. Amyloid-beta42 alters apolipoprotein E solubility in brains of mice with five familial AD mutations. J. Neurosci. Methods 196, 51-59 (2011)

51. Wu, Z., Guo, Z., Gearing, M. \& Chen, G. Tonic inhibition in dentate gyrus impairs long-term potentiation and memory in an Alzheimer's disease model. Nat. Commun. 5, 4159 (2014).

52. Tanzi, R. E. The synaptic Abeta hypothesis of Alzheimer disease. Nat. Neurosci. 8, 977-979 (2005).

53. Golde, T. E. The Abeta hypothesis: leading us to rationally-designed therapeutic strategies for the treatment or prevention of Alzheimer disease. Brain Pathol. 15, 84-87 (2005).

54. Weitz, T. M. \& Town, T. Microglia in Alzheimer's disease: it's all about context. Int. J. Alzheimer's Dis. 2012, 314185 (2012).

55. Heneka, M. T., Golenbock, D. T. \& Latz, E. Innate immunity in Alzheimer's disease. Nat. Immunol. 16, 229-236 (2015).

56. Ulrich, J. D. et al. Altered microglial response to Abeta plaques in APPPS1-21 mice heterozygous for TREM2. Mol. Neurodegener. 9, 20 (2014).

57. Lee, C. Y. D. et al. Elevated TREM 2 gene dosage reprograms microglia responsivity and ameliorates pathological phenotypes in Alzheimer's disease models. Neuron 97, 1032-1048 e1035 (2018).

58. Keren-Shaul, H. et al. A unique microglia type associated with restricting development of Alzheimer's disease. Cell 169, 1276-1290 e1217 (2017).

59. Krasemann, S. et al. The TREM2-APOE pathway drives the transcriptional phenotype of dysfunctional microglia in neurodegenerative diseases. Immunity 47, 566-581 e569 (2017).

60. Parkhurst, C. N. et al. Microglia promote learning-dependent synapse formation through brain-derived neurotrophic factor. Cell 155, 1596-1609 (2013).

61. Stellwagen, D., Beattie, E. C., Seo, J. Y. \& Malenka, R. C. Differential regulation of AMPA receptor and GABA receptor trafficking by tumor necrosis factoralpha. J. Neurosci. 25, 3219-3228 (2005).

62. Viviani, B. et al. Interleukin-1beta enhances NMDA receptor-mediated intracellular calcium increase through activation of the Src family of kinases. J. Neurosci. 23, 8692-8700 (2003).

63. Kawasaki, Y., Zhang, L., Cheng, J. K. \& Ji, R. R. Cytokine mechanisms of central sensitization: distinct and overlapping role of interleukin-1beta, interleukin-6, and tumor necrosis factor-alpha in regulating synaptic and neuronal activity in the superficial spinal cord. J. Neurosci. 28, 5189-5194 (2008).

64. Liu, C. C. et al. Neuronal heparan sulfates promote amyloid pathology by modulating brain amyloid-beta clearance and aggregation in Alzheimer's disease. Sci. Transl. Med. 8, 332ra344 (2016).

65. $\mathrm{Xu}, \mathrm{H}$. et al. Estrogen reduces neuronal generation of Alzheimer beta-amyloid peptides. Nat. Med. 4, 447-451 (1998).

66. Heneka, M. T. et al. NLRP3 is activated in Alzheimer's disease and contributes to pathology in APP/PS1 mice. Nature 493, 674-678 (2013).

67. Wen, L. et al. VPS35 haploinsufficiency increases Alzheimer's disease neuropathology. J. Cell Biol. 195, 765-779 (2011).

68. Sun, H. \& Neugebauer, V. mGluR1, but not mGluR5, activates feed-forward inhibition in the medial prefrontal cortex to impair decision making. J. Neurophysiol. 106, 960-973 (2011).

\section{Acknowledgements}

Research by the authors was supported by grants from the National Natural Science Foundation of China 81370459, 31400914 (to X.C.), 81701079 (to L.Z.), 81373999, 81774377 (to L.W.), and 81601227 (to R.Z.), grants from the Natural Science Foundation 
of Guangdong Province 2016A030306005 (to X.C.), 2016A030310371 (to R.Z.), grants from the Fundamental Research Funds for the Central Universities 20720180055

(to X.C.), grants from the Alzheimer's Association AARG-18-56635 (to X.C.), and C4C15-369446 (to H.X.). NIH grants RF1AG056130 (to G.B. and H.X.), R01AG035355 (to G.B.), R37AG027924 (to G.B.), and RF1AG056114 (to H.X.), grants from the Postdoctoral Science Foundation of China 2016M600503 and 2017T100469 (to L.Z.), a grant from the Tanz Family Funds (to H.X.), and a grant from the Natural Science Foundation of Fujian Province 2016J05203 (to R.Z.). X.C. also received funding from the Shenzhen Basic Research Program JCYJ20170818140904167.

\section{Author contributions}

X.C., G.B., and Li Zhong designed the project and wrote the paper; Li Zhong and Y.X. performed all the experiments and data analyses unless otherwise indicated; T.W. and R.H. performed mouse behavioral test; Y.X. and Y.Z. purified the sTREM2 protein; R.Z., Y.G., X.S., and Lin Zhu performed the electrophysiological experiment; Li Zhong, K.W., and D.W. performed the FACS experiment; K.W., K.C., N.W., and D.C. isolated and cultured primary microglia; Li Zhong, Y.M., M.S., and C.L. set up and performed sTREM2 ELISA; H.X., L.W., H.S., and D.D. reviewed the paper. All the authors read and approved the final manuscript.

\section{Additional information}

Supplementary Information accompanies this paper at https://doi.org/10.1038/s41467019-09118-9.

Competing interests: The authors declare no competing interests.
Reprints and permission information is available online at http://npg.nature.com/ reprintsandpermissions/

Journal peer review information: Nature Communications thanks Li Gan, Michela Matteoli, and the other anonymous reviewer(s) for their contribution to the peer review of this work.

Publisher's note: Springer Nature remains neutral with regard to jurisdictional claims in published maps and institutional affiliations.

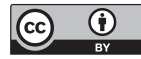

Open Access This article is licensed under a Creative Commons Attribution 4.0 International License, which permits use, sharing, adaptation, distribution and reproduction in any medium or format, as long as you give appropriate credit to the original author(s) and the source, provide a link to the Creative Commons license, and indicate if changes were made. The images or other third party material in this article are included in the article's Creative Commons license, unless indicated otherwise in a credit line to the material. If material is not included in the article's Creative Commons license and your intended use is not permitted by statutory regulation or exceeds the permitted use, you will need to obtain permission directly from the copyright holder. To view a copy of this license, visit http://creativecommons.org/ licenses/by/4.0/.

(C) The Author(s) 2019 